\title{
Evaluación del desempeño de sistemas de ventilación en salones de clase: Estudio numérico en edificios universitarios en Panamá
}

\author{
Performance Evaluation of Ventilation Systems in Classrooms: Numerical Study in \\ University Buildings in Panama
}

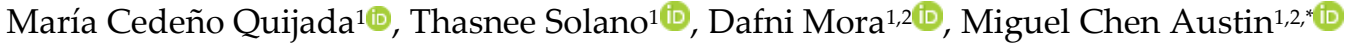 \\ ${ }^{1}$ Grupo de Investigación Energética y Confort en Edificaciones Bioclimáticas (ECEB), Facultad de Ingeniería Mecánica, Universidad \\ Tecnológica de Panamá, Ciudad de Panamá, Panamá; 0819-07289; maria.cedeno5@utp.ac.pa; thasneesolano@hotmail.com; \\ dafni.mora@utp.ac.pa \\ ${ }^{2}$ Centro de Estudios Multidisciplinarios en Ciencias, Ingeniería y Tecnología (CEMCIT-AIP), Ciudad de Panamá, Panamá; 0819- \\ 07289
}

Citación: Cedeño, M., Solano, T, Mora, D., \& Chen, M., (2022). Evaluación del desempeño de sistemas de ventilación en salones de clase: Estudio numérico en edificios universitarios en Panamá. Novasinergia. 5(1). 100-127. https://doi.org/10.37135/ns.01.09.07

Recibido: 27 septiembre 2021

Aceptado: 29 enero 2022

Publicado: 31 enero 2022

Novasinergia

ISSN: 2631-2654

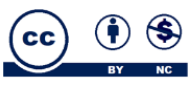

Copyright:

Este es un artículo de acceso abierto distribuido bajo los términos $\mathrm{y}$ condiciones de una licencia de Creative Commons Attribution (CC BY NC).

(http://creativecommons.org/licens es/by/4.0/).
*Correspondencia: miguel.chen@utp.ac.pa

Resumen: La calidad del aire juega un papel decisivo en el rendimiento de los ocupantes de espacios interiores; además, se estima que un $30 \%$ de la vida de una persona corresponde a su formación académica. El objetivo de esta investigación fue determinar si los sistemas de ventilación de los edificios de la Universidad Tecnológica de Panamá proveen una calidad de aire apropiada para sus alumnos, mediante la evaluación del desempeño de la ventilación en modo pasivo y mecánico; se utilizó la interfaz dinámica DesignBuilder para simular los casos de estudio. Los indicadores utilizados fueron la concentración de $\mathrm{CO}_{2}$, las tasas de renovación de aire interior y el confort térmico. Para esto se tomaron como referencia los requerimientos mínimos de la normativa panameña y normativas internacionales. Los resultados demostraron que el uso de ventilación natural resulta insuficiente debido a la inconformidad térmica y altas concentraciones de $\mathrm{CO}_{2}$. Por el contrario, el uso de ventilación mecánica mejoró los niveles de confort térmico pero la concentración de $\mathrm{CO}_{2}$ se mantuvo ligeramente fuera de límites aceptables. Estos resultados demostraron que los edificios no están diseñados para operar en modo pasivo, lo que restringe su operación exclusivamente en modo mecánico.

Palabras clave: Calidad del aire, confort térmico, dióxido de carbono, edificios, renovación de aire, simulación dinámica, ventilación mecánica, ventilación natural.

Abstract: Air quality plays a decisive role in the performance of the occupants
of indoor spaces; it is estimated that 30\% of a person's life corresponds to their
academic training. This research aimed to determine if the ventilation systems
in the Technological University of Panama buildings provide appropriate air
quality for its students. We evaluated the ventilation performance in passive
and mechanical mode; the dynamic interface DesignBuilder simulated case
studies. The indicators used were CO2 concentration, indoor air renewal rates,
and thermal comfort. The minimum requirements of Panamanian and
international standards were used as a reference. The results showed
insufficient natural ventilation due to thermal discomfort and high CO2
concentrations. In contrast, mechanical ventilation improved thermal comfort
levels, but the CO2 concentration remained slightly outside acceptable limits.
These results demonstrated that the buildings are not designed to operate
passively, restricting their operation exclusively in mechanical mode.

Keywords: Air renewal, buildings, carbon dioxide, dynamic simulation, indoor air quality, mechanical ventilation, natural ventilation, thermal comfort. 


\section{Introducción}

Asegurar una calidad de aire interior óptima en edificios construidos en los últimos 30 años, se ha convertido en un objetivo primordial en cuanto a confort térmico y desempeño de ventilación se refiere (Vartires et al., 2018). Solemos estar el 90\% de nuestras vidas dentro de ambientes interiores, desde nuestros hogares, colegios, universidad, trabajo, entre otras actividades (Fantozzi $\mathcal{E}$ Rocca, 2020) y de ese porcentaje, se estima que un $30 \%$ corresponde al tiempo dedicado a colegiatura y educación superior (Bakó-Biró, Clements-Croome, Kochhar, Awbi \& Williams, 2012; Wargocki, Porras-Salazar, Contreras-Espinoza $\mathcal{E}$ Bahnfleth, 2020), por lo que asegurar una buena calidad del aire interior, es fundamental.

La calidad del aire interior (del inglés "Indoor Air Quality" o IAQ), según la institución American Society of Heating, Refrigerating and Air-Conditioning Engineers (ASHRAE) se define como el aire dentro de un edificio o estructura que no contiene contaminantes en concentraciones peligrosas para las personas (ASHRAE, 2009, 2019). Los contaminantes son agentes externos, tales como partículas de polvo, gérmenes, concentración de gases, compuestos orgánicos volátiles, entre otros (Cruz et al., 2020). Las concentraciones aceptadas para un alto IAQ, son determinadas por autoridades locales según el país donde se evalúe y también, por regulaciones internacionales, cuyo objetivo radica en que la mayoría de las personas expuestas ( $80 \%$ o más) no expresen inconformidad o insatisfacción dentro un espacio (ASHRAE, 2020). Si este porcentaje resulta menor, se refleja en una baja calidad del aire ante la presencia de contaminantes indeseables, por lo que es necesario considerar diversas técnicas para la purificación del aire: si el contaminante son partículas (polvo), pueden eliminarse por filtración y aquellos en forma de gases u olores, mediante un nivel aceptable de ventilación (Pita, 1994).

La ventilación, implica el reemplazo continuo del aire interior potencialmente contaminado y usualmente es definida paramétricamente como la tasa de cambio de aire o renovación de aire (también conocida en inglés como "Air Change Rate" o ACH) y puede ser natural, mecánica e híbrida. La ventilación natural, también conocida como ventilación en modo pasivo, es aquella en la que la renovación del aire se produce exclusivamente por la acción del viento o por la existencia de un gradiente de temperatura entre el punto de entrada y salida de un espacio (Jiménez, 2021); la ventilación mecánica, es aquella en la que la renovación del aire se produce por el funcionamiento de aparatos electromecánicos con extracción mecánica equilibrada. A este tipo de ventilación se le refiere como ventilación o modo activo. Finalmente, la ventilación híbrida o mixta, es aquella en la que cuando las condiciones de presión y temperatura ambientales son favorables, la renovación del aire se produce en modo pasivo y cuando son desfavorables, se utilizan equipos electromecánicos en modo activo (Jiménez, 2021).

El aire interior, es uno de los factores que determina la funcionalidad y economía de un edificio debido a que afecta directamente a las personas incidiendo en su capacidad de realizar actividades. Tanto la ventilación como las tasas de renovación del aire interior juegan un papel decisivo en cuanto a la calidad del aire ofrecida a los ocupantes de un recinto; en los nuevos edificios se producen emanaciones de materiales y elementos, los que, sumados a una limpieza y mantenimiento no 
periódicos, contribuyen a la contaminación del aire interior y constituyen el denominado "Síndrome del Edificio Enfermo" (Sick Building Syndrome “SBS") (Jiménez, 2021).

El término de SBS, es utilizado para describir situaciones en la que los ocupantes de un espacio cerrado experimentan efectos agudos que afectan su salud y confort. Estos efectos van ligados al tiempo que pasan dentro de un edificio o estructura, pero no se puede identificar ninguna causa o enfermedad específica, solo los síntomas: dolor de cabeza, irritación en los ojos, nariz o garganta, tos seca, piel irritada, fatiga, náusea e incluso dificultad para concentrarse. La mayoría de estos síntomas desaparecen al salir del edificio. Las quejas pueden estar localizadas en una habitación o zona en particular, o pueden estar muy extendidas por todo el edificio (United States Environmental Protection Agency (EPA), 1991). Algunas de las causas del SBS, constituye la presencia de contaminantes proveniente de fuentes dentro de los edificios o de fuentes externas, contaminantes biológicos, químicos y ventilación inadecuada. Este síndrome puede afectar desde edificios gubernamentales, oficinas y espacios educativos (Ma'bdeh, Al-Zghoul, Alradaideh, Batanineh $\mathcal{E}$ Ahmad, 2020).

William (2017) después de recopilar 26 estudios a nivel global relacionados con la ventilación en espacios escolares, afirmó que las tasas de ventilación en salones de clase, usualmente son más bajas de lo sugerido en estándares internacionales como ASHRAE (2019), D'Ambrosio et al. (2010) y European Committee for Standarization (2006). Para evaluar el desempeño del IAQ, se utiliza las tasas de renovación de aire interior $(\mathrm{ACH})$ pero entre los principales indicadores, también está el nivel de dióxido de carbono ya que este gas se utiliza como referencia en espacios donde la principal fuente de contaminación es el metabolismo humano (Batterman et al., 2017; Calama-González, LeónRodríguez \& Suárez, 2019; Cruz et al., 2020) . Una tasa de renovación de aire mínima de 3.0 ACH ó 2 a $3.5 \mathrm{~L} / \mathrm{s}^{*}$ persona y una máxima concentración de dióxido de carbono de 1000 ppm (ASHRAE, 2019) son los valores de los indicadores utilizados como referencia.

Vartires et al. (2018), mediante un estudio experimental compararon dos escenarios de ventilación: un aula con ventilación natural y un aula que utilizaba ventilación híbrida. En el aula con ventilación natural, los niveles de $\mathrm{CO}_{2}$, superaban los 2500 ppm, contrario al salón con ventilación híbrida que el máximo nivel de $\mathrm{CO}_{2}$ fue de 1200 ppm. De forma semejante Simanic, Nordquist, Bagge $\mathcal{E}$ Johansson (2019) realizaron un estudio exploratorio en siete colegios de Suecia. Los autores resaltaron que el uso de equipos modernos de acondicionamiento de aire, son necesarios para proveer condiciones ambientales favorables para los estudiantes. A diferencia de Vartires et al. (2018), los autores indican que los estudiantes mantuvieron satisfacción y confort en concentraciones aproximadas de $600 \mathrm{ppm}$.

Calama-González et al. (2019) indicaron que la ocupación tiene relación con las condiciones ambientales. Su estudio realizado en una escuela ubicada en la zona mediterránea de España indicó que durante períodos desocupados con el uso de ventilación natural (ventanas totalmente abiertas), los niveles de $\mathrm{CO}_{2}$ estaban por debajo de $425 \mathrm{ppm}$. En períodos de $45 \%$ de ocupación, los niveles se encontraban entre 500 a 1000 ppm y en ocupación total, los niveles superaban los 1500 ppm. A pesar de mantener los niveles no tan excesivamente altos y que las aulas contaban con mecanismos de 
ventilación natural, los niveles de $\mathrm{CO}_{2}$ justificaban la necesidad de instalar sistemas de ventilación mecánica.

Adicionalmente, estudios anteriores (Fisk, 2017; Pulimeno, Piscitelli, Colazzo, Colao $\mathcal{E}$ Miani, 2020; Simanic et al., 2019; Wargocki et al., 2020) señalaron que una pobre ventilación además de afectar la salud de los estudiantes, puede disminuir el rendimiento académico. En este sentido, Wargocki et al. (2020) realizaron un estudio experimental en un colegio de estudiantes no mayores de 18 años, en el que buscó establecer la relación entre la calidad del aire en aulas y el desempeño de los estudiantes en colegios cuyas instalaciones utilizaban ventilación mecánica. La calidad del aire interior se caracterizó por la concentración de $\mathrm{CO}_{2}$ y las tasas de renovación de aire interior; el desempeño académico, fue evaluado por pruebas psicológicas que miden habilidades y destrezas cognitivas. Inicialmente, (ASHRAE Epidemic Task Force, 2020) los valores registrados de $\mathrm{CO}_{2}$ oscilaban desde 600 ppm a 4300 ppm y las tasas de renovación de aire se mantuvieron en 2.0 $\mathrm{L} / \mathrm{s}^{*}$ persona. Al aumentar la tasa de ventilación a $10 \mathrm{~L} / \mathrm{s}^{*}$ persona y disminuir la concentración de dióxido de carbono a 900 ppm, hubo un aumento de productividad de 12\%. También, hubo mayor asistencia y mejorías de desempeño en las funciones de los alumnos.

Evaluar el desempeño de la ventilación durante la pandemia SARS COVID-19 resulta de interés puesto que es conocido, que algunas enfermedades se propagan por aerosoles infecciosos (The American Institute of Architects (AIA), 2021). El riesgo de propagación de patógenos y el número de personas expuestas puede verse afectado sea positiva o negativamente por el aire interior de recintos ocupados y por los sistemas de acondicionamiento de aire o extracción local (ASHRAE Epidemic Task Force, 2020). La eficacia de la ventilación se debe evaluar para reducir el riesgo de transmisión de enfermedades, con este fin Ascione, De Masi, Mastellone \& Vanoli (2021), publicaron un estudio numérico relacionado al "diseño de aulas seguras en espacios educativos en fin de enfrentar contagios y transmisión de enfermedades" utilizando dinámica de fluidos computacional (CFD). Este tipo de programación (Hu, 2012) es utilizada para simular, analizar y resolver programas que implican flujos de fluidos, como el aire. El autor indicó que se recomienda un mínimo de 6.5 a 7.9 ACH para mantener una alta calidad de aire interior y un riesgo mínimo de infección de enfermedades respiratorias.

Hasta la fecha, son pocos los estudios relacionados al desempeño de la ventilación en ambientes universitarios y escolares en Latinoamérica. En algunos países tales como Costa Rica (Ministerio de Salud de Costa Rica, 2021), indican que toman como referencia los estándares ASHRAE (2009. 2019) para el diseño referente a la ventilación en edificios y otros, como el caso de Chile, no cuentan con normas que regulen el confort ambiental o ventilación en edificios escolares (Trebilcoc, Soto-Muñoz, Figueroa $\mathcal{E}$ Piderit-Moreno, 2016) solo cuentan con el Decreto Supremo 548 (Ministerio de Educación de Chile, 1989), que indica una tasa de ventilación de 2 ACH cuando las aulas proveen ventilación mecánica.

Los estudios anteriores, muestran la importancia de un diseño de aire acondicionado y ventilación apropiados para espacios educativos. El indicador más utilizado para evaluar el desempeño de la ventilación es la tasa de renovación de aire. Sin embargo, incluso cuando para determinar la calidad del aire se determina la concentración de particulado $\mathrm{PM}_{10}, \mathrm{PM}_{2.5}$, compuestos volátiles orgánicos 
(VOCs) y aldehídos, la concentración de dióxido de carbono, resulta ser un indicador útil para definir la ocupación en espacios interiores y controlar la calidad de aire ambiental.

Además, es importante resaltar que, países como China (Sun, Wang, Zhang $\mathcal{E}$ Sundell, 2011), Suecia (Simanic et al., 2019), Escocia (Wargocki et al., 2020) e Italia (Ascione et al., 2021) pese a que tienen normativas propias para el diseño de acondicionamiento del aire y ventilación, utilizan los estándares americanos (ASHRAE, 2009, 2019, 2020) como referencia para el diseño y evaluación del desempeño de la ventilación.

En el caso de Panamá, se utiliza el Reglamento de Aire Acondicionado y Ventilación conocido como "RAV" (Junta Técnica de Ingeniería y Arquitectura (JTIA), \& Asociación Panameña de Aire Acondicionado y Ventilación (APAYRE)., 2014). En este reglamento, se recalca un mínimo de 5 $\mathrm{L} / \mathrm{s}^{*}$ persona en aulas educativas.

Evaluar el desempeño de la ventilación considerando exclusivamente las tasas de renovación de aire, resulta insuficiente al no tener control sobre los contaminantes presentes en el aire y el confort térmico de los usuarios; por esta razón, el objetivo de esta investigación consiste en evaluar de forma cuantitativa el desempeño de la ventilación en modo pasivo y modo activo (natural y mecánica) en edificios universitarios en términos de la tasa de renovación interior, concentración de $\mathrm{CO}_{2}$ y considerar al mismo tiempo, el confort térmico de los estudiantes de la Universidad Tecnológica de Panamá. El confort térmico es definido por (ASHRAE, 2020) como la condición en donde una persona expresa satisfacción con el entorno térmico. Está condicionada por factores ambientales como la temperatura del aire, velocidad del aire, humedad y por factores personales como la ropa y el calor metabólico. Wargocki $\mathcal{E}$ Wyon (2013) concluyeron que tanto el confort térmico como la calidad del aire en las aulas, son factores importantes en el proceso de aprendizaje. La motivación de esta investigación surge a raíz de la escasa literatura encontrada en casos aplicados en América Latina y comprobar si Panamá, cumple con los mínimos requeridos de la normativa internacional (ASHRAE, 2020).

\section{Metodología}

La metodología implementada para la evaluación del desempeño de la ventilación de los edificios universitarios fue de carácter cuantitativo. Se utilizó el software de simulación dinámica de Energy Plus: DesignBuilder ya que esta herramienta, permite predecir resultados de tasas de ventilación, temperatura interior, humedad, consumo de energía, confort térmico (entre otros) en horarios de ocupación establecidos (Trebilcock et al., 2016). La flexibilidad y precisión en predicciones es posible mediante el uso de métodos numéricos avanzados, como CFD, que utiliza este software. La simulación fue realizada considerando un año lectivo completo (12 meses).

\subsection{Descripción del caso de estudio}

Se estudiaron dos de los edificios que componen al Campus Víctor Levi Sasso conocidos como Edificio $\mathrm{N}^{\circ} 1$ (E1) y Edificio $\mathrm{N}^{\circ} 5$ (E5). El E1 incluye las aulas y oficinas de la Facultad de Ingeniería Civil, Eléctrica, Mecánica e Industrial y el E5, exclusivamente con salones de clases. 


\section{Localización Geográfica}

La Universidad Tecnológica de Panamá, es una universidad estatal con sede central ubicada en la vía Centenario del corregimiento de Ancón, Distrito de Panamá, Ciudad de Panamá (coordenadas 901'25" Norte, 79³1'53" Oeste). El Campus Víctor Levi Sasso, es la sede central en el país. En la figura 1, se aprecian los dos edificios evaluados, E5 a la izquierda y el E1 derecha con sus alrededores.

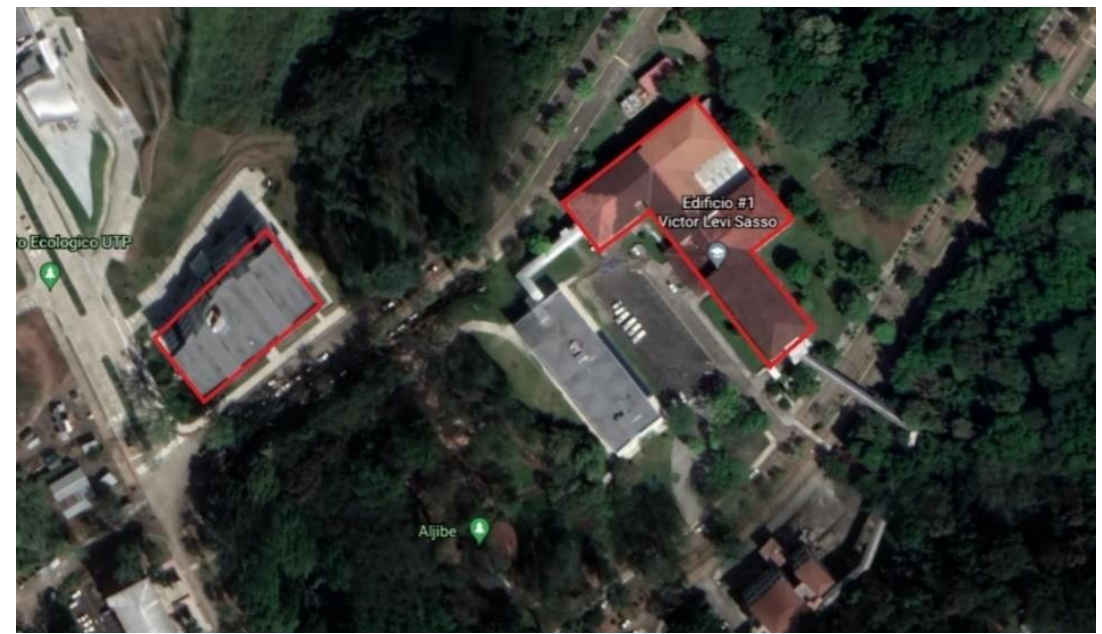

Figura 1: Ubicación del edificio No. 5 (izquierda) y edificio No. 1 de la Universidad Tecnológica de Panamá (Google Earth, s.f.).

Para el caso de estudio, se seleccionaron salones de clases en ambos edificios. En el caso del E5, se seleccionaron dos aulas centrales (con una sola ventana) y dos aulas de esquinas (con dos ventanas) de la planta baja y tercer piso del edificio. Estas aulas fueron seleccionadas debido a que mantienen el mismo tamaño y ubicación en las plantas antes mencionadas (Figura 2, cuadrados rojos).
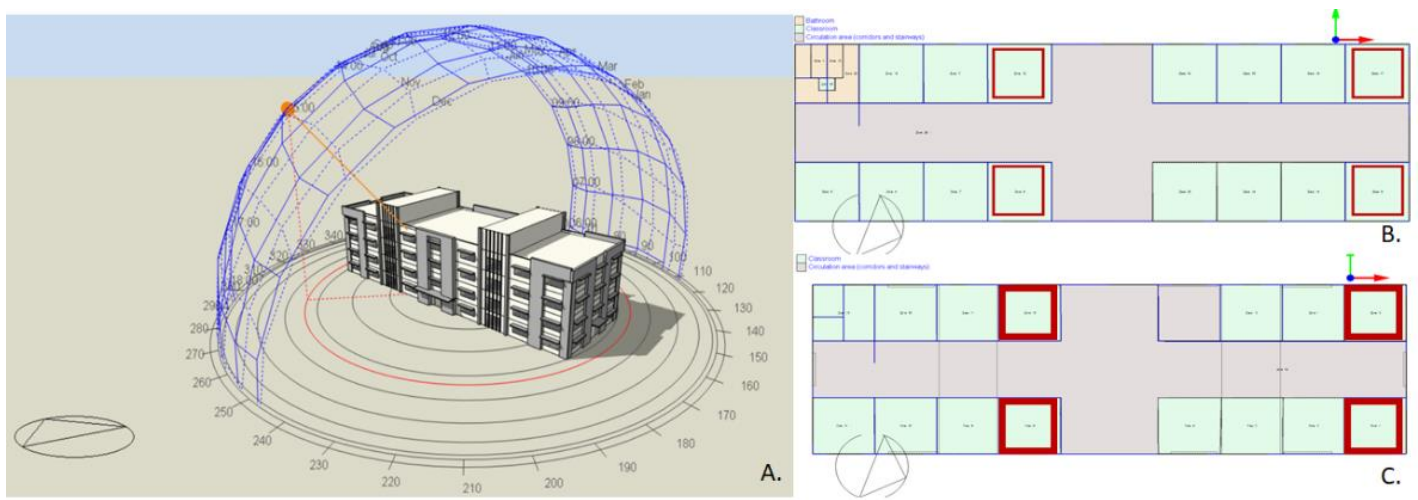

Figura 2: (A). Vista 3D del E5 en DesignBuilder, con el norte señalado, (B) Vista de planta de la planta baja y (C) Vista de planta del tercer piso.

Igual que en el E5, para el E1 se seleccionaron aulas de la planta baja y el tercer piso, como se señala en la figura 3. Sin embargo, entre plantas del E1, la distribución de salones puede variar debido a remodelaciones realizadas por los administrativos de cada facultad, donde incluyeron oficinas administrativas y ampliaron salones de clase por lo que se escogieron aulas con características similares. Dado que el E1 tiene una población estudiantil superior, se escogieron siete salones: dos del ala de salones (parte inferior de la Figura 3B y 3C), una del ala de laboratorio central, dos del ala de laboratorios frontal y dos del ala posterior (parte superior de la Figura 3B y 3C). 


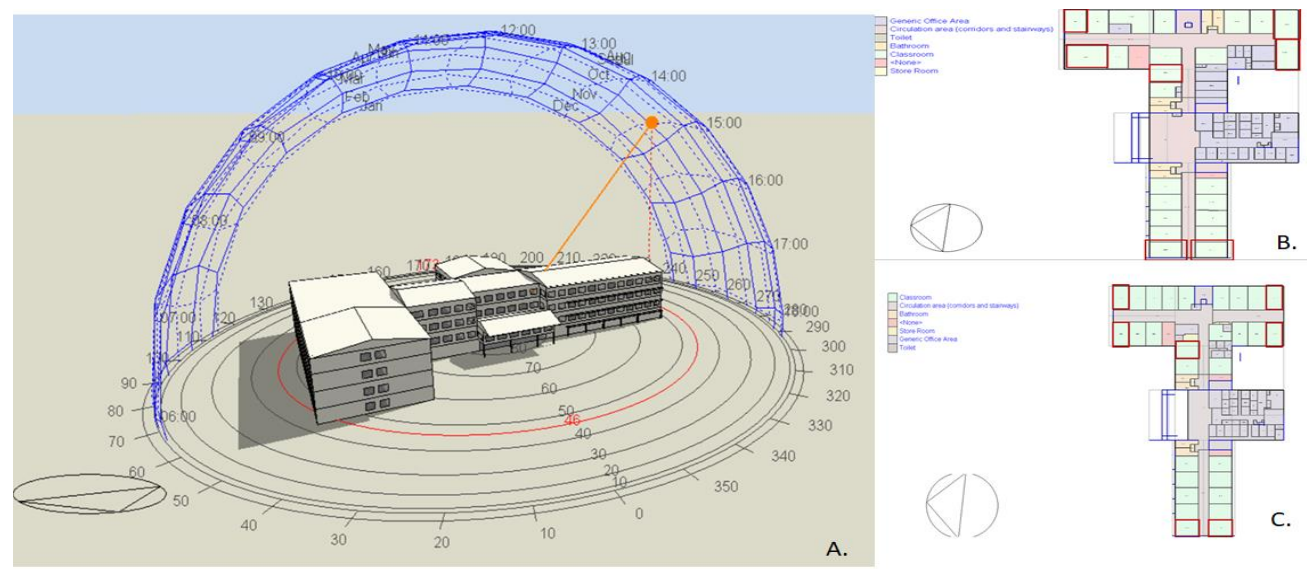

Figura 3: (A). Vista 3D del E1 en DesignBuilder, con el Norte señalado, (B) Vista de planta de la planta baja y (C)Vista de planta del tercer piso.

\section{Detalles de construcción}

Los materiales que componen la envolvente del E1 y E5, son listados en la tabla 1 como también sus valores de transmitancia dados en $\left(\mathrm{W} / \mathrm{m}^{2} \mathrm{~K}\right)$. Estos datos fueron útiles para la simulación dinámica con el software, DesignBuilder.

Tabla 1: Detalles de construcción; E1 y E5 de la Universidad Tecnológica de Panamá*.

\begin{tabular}{ccc}
$\begin{array}{c}\text { Componentes de la } \\
\text { envolvente }\end{array}$ & Material & $\begin{array}{c}\text { Coeficiente general de } \\
\text { transferencia de calor } \mathrm{U}\left(\mathrm{W} / \mathrm{m}^{2} \mathrm{~K}\right)\end{array}$ \\
\hline
\end{tabular}

Paredes (exteriores e Bloque de concreto de $0.100 \mathrm{~m}$ de espesor con doble interiores) repello liso de $0.015 \mathrm{~m}$ de espesor.

1.94

Suelos

Baldosa de porcelanato $0.040 \mathrm{~m} \times 0.040 \mathrm{~m}$

Tipo emparedado de $0.038 \mathrm{~m}$ de espesor con poliuretano expandido, sobre estructura de metal.

Techo Suspendido. Fibra de mineral de $0.0508 \mathrm{~m} \times 0.0508 \mathrm{~m}$ en tes de aluminio a $2.700 \mathrm{~m}$ de piso acabado. Madera de pino $2.00 \mathrm{~m} \times 1.00 \mathrm{~m}$

Cielo Raso

Puertas

Ventanas Corredizas, acristalamiento sencillo tintado gris con $0.006 \mathrm{~m}$ de espesor

*Datos proporcionados por la Dirección General de Ingeniería y Arquitectura de la Universidad Tecnológica de Panamá.

Detalles sobre el uso de la energía

Similarmente, el uso de energía tanto de equipos como sistema de aire acondicionado, así como también el horario de funcionamiento, es señalado en la tabla 2. Estos datos también se incluyeron en la simulación dinámica realizada en el software DesignBuilder.

Tabla 2: Detalles del uso de energía y horarios de funcionamiento

\begin{tabular}{cclc} 
Equipos & Consumo estimado & \multicolumn{1}{c}{ Horario de funcionamiento } \\
\hline Iluminación general interior (bombillos & $32 \mathrm{~W}$ & Lunes a viernes & $07: 00-22: 30$ \\
fluorescentes) & & Sábados & $08: 00-18: 00$ \\
& & Lunes a viernes & $07: 00-22: 30$ \\
Densidad de consumo por equipos & $17.6 \mathrm{~W} / \mathrm{m}^{2}$ & Sábados & $18: 00-18: 00$ \\
Iluminación general exterior & $100 \mathrm{~W}$ & Lunes a domingo & $07: 00$ \\
Sistema de acondicionamiento de aire (Chiller & \multirow{2}{*}{$30 \mathrm{kWh} / \mathrm{m}^{2}$} & Lunes a viernes &
\end{tabular}


Detalles de ocupación

Un total de 3255 estudiantes constituye la población del E1 mientras que, en el E5, 2206. Referente a la vestimenta se utilizó un valor de 0.5 clo. El clo, hace referencia a la unidad de aislamiento térmico proporcionada por las prendas de vestir. Se especificó un nivel de actividad de 0.90 met. El met es la tasa de transformación de energía química en calor y energía mecánica producto de las actividades metabólicas de una persona (ASHRAE, 2020).

Tanto los detalles de la ocupación y el horario de asistencia fueron señalados en el software de simulación dinámica, DesignBuilder.

\subsection{Estrategia de evaluación del sistema de ventilación}

Para iniciar, se determinó el valor mínimo de ventilación requerido ASHRAE $(2009,2019)$. Esta normativa, establece en la ecuación (1) el mínimo de ventilación en una zona respiratoria que constituya una edificación con ventilación natural, mecánica o híbrida. En este caso, indica el nivel mínimo de ACH que las aulas del E1 y E5 deben cumplir tanto en modo pasivo como en modo activo para asegurar una alta calidad de aire interior. El valor determinado con esta ecuación fue útil para evaluar los resultados obtenidos con DesignBuilder. La ecuación (1) se formula de la siguiente manera:

$$
V=R_{p} P_{z}+R_{a} A_{z}
$$

Donde, $\mathrm{V}$ representa el mínimo de ventilación requerida en una zona respiratoria, en este caso, sería el mínimo de ventilación en las aulas de clase de la Universidad Tecnológica de Panamá. $R_{p}$ es la tasa de renovación mínima para salones de clases $\left(5 \mathrm{~L} / \mathrm{s}^{*}\right.$ persona) indicado por el estándar 62.1 (ASHRAE, 2009, 2019). P es la cantidad de estudiantes por aula y $R_{a}$ es la tasa de renovación mínima por unidad de área, también señalado por ASHRAE (2019), $0.9 \mathrm{~L} / \mathrm{s}^{*} \mathrm{~m}^{2}$. Finalmente, $\mathrm{A}_{z}$ es el área del salón de clases.

De los valores antes mencionados, varían $\mathrm{A}_{\mathrm{z}} \mathrm{y} \mathrm{P}_{\mathrm{z}}$, siendo el área de cada aula y el total de la población estudiantil en cada edificio.

Para el E5, el área promedio de salones ronda los $48.88 \mathrm{~m}^{2}$ y el total de estudiantes es 2206 estudiantes. Mientras que, en el E1, el área promedio de salones ronda los $60.70 \mathrm{~m}^{2}$ y su población total es de 3255 estudiantes.

Siguiendo esta ecuación, las tasas de renovación de aire mínima para modo pasivo y modo activo, se señalan en la tabla 3:

Tabla 3: Mínimo de ACH para E1 y E5 de la Universidad Tecnológica de Panamá.

\begin{tabular}{cc}
\hline Edificio & $\begin{array}{c}\text { Tasas de renovación de } \\
\text { aire estimadas }(\mathrm{ACH})\end{array}$ \\
\hline $\mathrm{N}^{\circ} 5$ & 5.13 \\
$\mathrm{~N}^{\circ} 1$ & 4.32 \\
\hline
\end{tabular}

Indicadores y criterios utilizados para la evaluación de la ventilación natural (modo pasivo)

Para el análisis del modo pasivo en ambos edificios, se estableció un horario de ocupación de lunes a viernes de 07:00 a 22:30 y sábados de 08:00 a 18:00. Este mismo horario fue utilizado para el 
uso de la energía exceptuando el sistema de acondicionamiento de aire. Las puertas y ventanas se mantuvieron $100 \%$ abiertas en dicho horario. Las luces externas se mantienen encendidas en un horario de 18:00 a 07:00.

Los parámetros considerados para evaluar la ventilación en ambos modos fueron categorizados como aquellos de calidad del aire interior y de confort térmico.

\section{Calidad del aire interior}

Se consideraron las tasas de renovación de aire interior y la concentración de $\mathrm{CO}_{2}$ en espacios interiores. Los valores considerados como válidos serán aquellos inferiores a 1000 ppm como lo establece el estándar 62.1 (ASHRAE, 2019).

\section{Confort térmico}

Niveles aceptables de confort térmico pueden ser medidos con el uso de los indicadores del voto medio previsto (PMV) o modelos adaptativos. El PMV es un índice que establece una predicción cuantitativa referente al porcentaje de personas insatisfechas con la sensación térmica de un recinto (ASHRAE, 2020). Este índice, va en una escala del -3 a +3 donde se señala las categorías de extremadamente frío (-3), frío (-2), ligeramente frío (-1), sensación neutral (0), ligeramente caliente $(+1)$, caliente $(+2)$ y muy caliente $(+3)$. Para el caso de estudio, se consideró la sensación neutral como confort térmico admisible.

Análogamente el modelo de Fanger adaptativo al 80\% de aceptabilidad, es un modelo empírico que relaciona la temperatura interior o rangos aceptables de temperatura con parámetros climatológicos (ASHRAE, 2020). Este índice se aplica en casos donde no se utilicen sistemas de acondicionamiento de aire (ventilación natural), los ocupantes son libres de adaptar su vestimenta a las condiciones climatológicas donde se encuentren (desde 0.5 a 1.0 clo), las tasas metabólicas se encuentran entre 1.0 a 1.5 met y también, la temperatura exterior debe ser menor a $33.5^{\circ} \mathrm{C}$ (ASHRAE, 2020). Si alguna de estas cuatro condiciones no cumple, el modelo adaptativo no es aplicable. Este parámetro se evalúa con valores desde " 1 " a " -1 ", donde un valor de " 1 " indica que la temperatura interior se encuentra dentro de los límites establecidos de confort térmico y los ocupantes se sienten cómodos térmicamente, mientras que un valor de " 0 " indica entonces que la temperatura se encuentra fuera del rango permitido y los ocupantes se encuentran insatisfechos (DesignBuilder Software, 2021); esto quiere decir que mientras más cercano al cero, más disconformidad térmica presentarán los estudiantes de los salones. En resultados obtenidos de " -1 ", la norma indica que son resultados "no aplicables" (ASHRAE , 2020). En este caso, interpretamos el valor de "1" como confort térmico admisible y "-1" como insatisfacción térmica.

\section{Indicadores y criterios utilizados para la evaluación de la ventilación mecánica: modo activo}

Para el análisis del modo mecánico en ambos edificios, se estableció un horario de ocupación de lunes a viernes de 07:00 a 22:30 y sábados de 08:00 a 18:00; este mismo horario fue utilizado para el uso de la energía, exceptuando las luces de exteriores que se mantienen encendidas de 18:00 a 07:00. El sistema de acondicionamiento de aire se utiliza en un horario de lunes a viernes de 07:00 a 
22:30 con puertas y ventanas siempre cerradas. Para la calidad del aire interior, se utilizaron como indicadores la concentración de $\mathrm{CO}_{2}$ y las tasas de renovación de aire interior y para el confort térmico, el PMV.

\section{Resultados}

Después de obtener las simulaciones en modo pasivo y activo de ambos edificios, los resultados fueron tabulados y posteriormente graficados para el análisis de los datos.

Sistema de ventilación natural, modo pasivo

Para los resultados del modo pasivo, se presentan los gráficos que ilustran la calidad del aire y confort térmico de los estudiantes. En términos de calidad del aire, se comparan las tasas de renovación interior con la concentración de $\mathrm{CO}_{2}$; con respecto a confort térmico, las tasas de renovación de aire interior fueron comparadas con el voto medio previsto y con el modelo adaptado al $80 \%$ de aceptabilidad térmica.

En la evaluación en modo pasivo, fue necesario distinguir los vientos predominantes de cada edificio. En las figuras 4 y 5, se observa la dirección de los vientos predominantes para cada estructura. La flecha negra, señala la posición norte de cada construcción mientras que la flecha roja señala la dirección de los vientos predominantes. Las flechas dentro de los edificios señalan el intercambio de vientos: la flecha roja ilustra el viento que atraviesa a los edificios y la azul, el viento que sale producto del intercambio de flujos de aire. En el E5, los vientos predominantes provienen del Noroeste (Figura 4), mientras que, en el E1, los vientos predominantes provienen del Noreste (Figura 5).

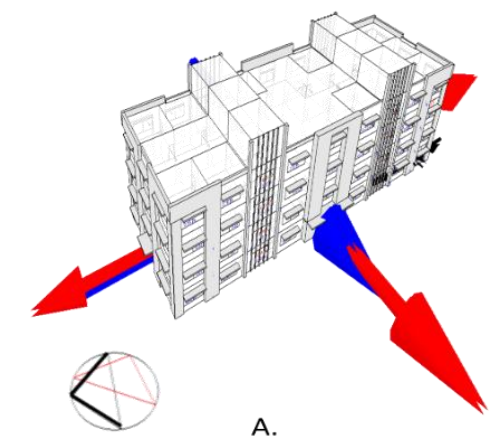

A.

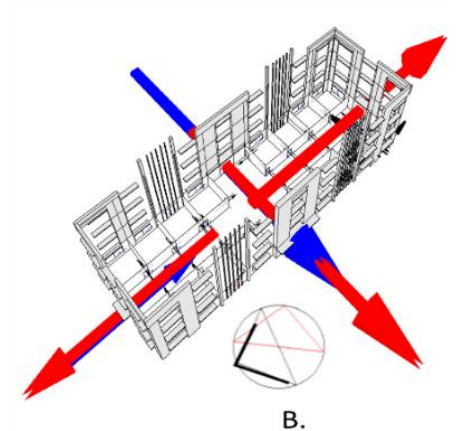

B.

Figura 4: (A) Dibujo 3D del E5 con los vientos predominantes en la zona durante los 12 meses del año, señalados en forma de vector y (B) Detalles del viento predominante dentro del edificio.

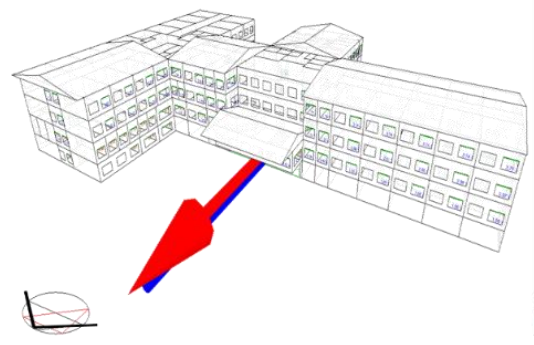

A.

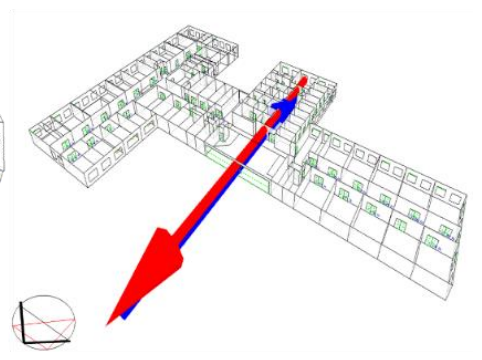

B.

Figura 5: (A) Dibujo 3D del E1 con los vientos predominantes en la zona durante los 12 meses del año, señalados en forma de vector y (B) Detalles del viento predominante dentro del edificio. 
Edificio No. 5: evaluación de la calidad del aire

La calidad del aire fue establecida con la concentración de $\mathrm{CO}_{2}$ en conjunto de las tasas de renovación del aire interior. Los resultados se detallan para la planta baja en la figura 6 y para el tercer piso, en la figura 7.

En el E5 los mayores valores de $\mathrm{CO}_{2}$ se obtuvieron en las aulas de la planta baja; superaron los 2000 ppm, el doble del límite establecido de 1000 ppm como se señala en la figura 6 (ASHRAE, 2009, 2019). En contraste los valores que se obtuvieron de las aulas del tercer piso registraron a lo largo del año concentraciones menores a 1000 ppm, siendo 600 ppm la concentración más alta en el mes de mayo. Particularmente, las aulas de las esquinas que cuentan con dos ventanas fueron las zonas donde el $\mathrm{CO}_{2}$ se mantenía por debajo de 1000 ppm a lo largo, tanto en la planta baja como el tercer piso. También, las aulas ubicadas en barlovento fueron aquellas que registraron mayor concentración de $\mathrm{CO}_{2}$ y menores tasas de renovación de aire.

En cuanto a las tasas de renovación de aire interior, en las aulas de la planta baja se obtuvieron tasas entre 1.3 y $1.45 \mathrm{ACH}$ y el tercer piso, de 1.15 a $1.3 \mathrm{ACH}$. Ambas plantas no cumplieron el mínimo calculado de $5.13 \mathrm{ACH}$ con la ecuación (1) para el E5. Al observar el comportamiento de ambos gráficos, se determina que las tasas de renovación de aire interior en modo pasivo van ligadas de forma inversamente proporcional al nivel de concentración de $\mathrm{CO}_{2}$.

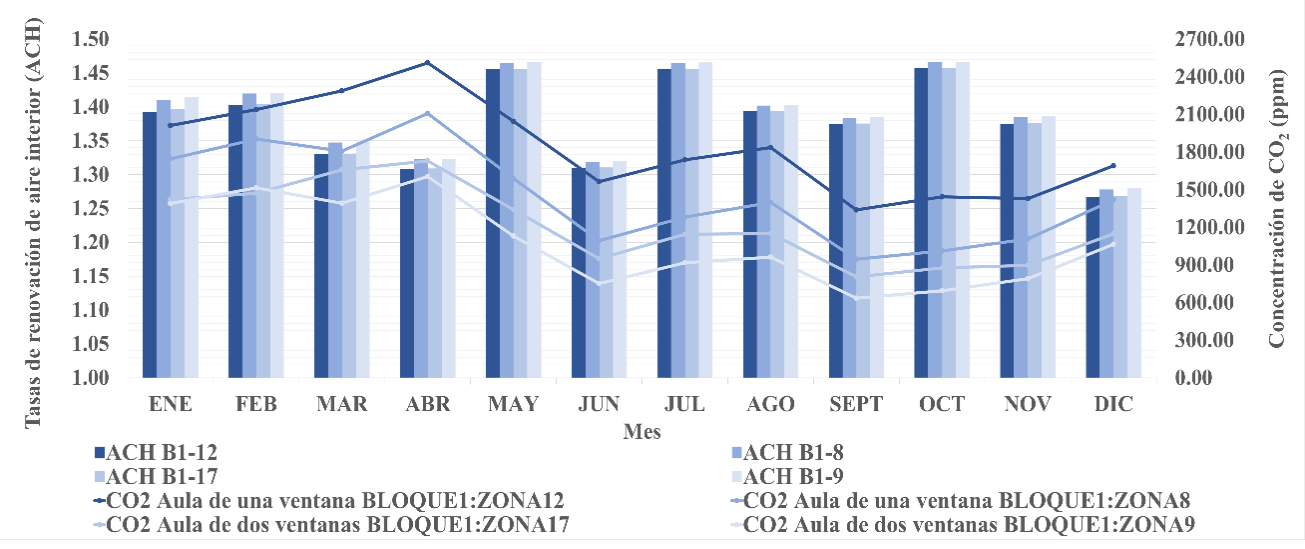

Figura 6: Comparación de tasas de renovación de aire interior con concentración de $\mathrm{CO}_{2}$. E5, planta baja, modo pasivo.

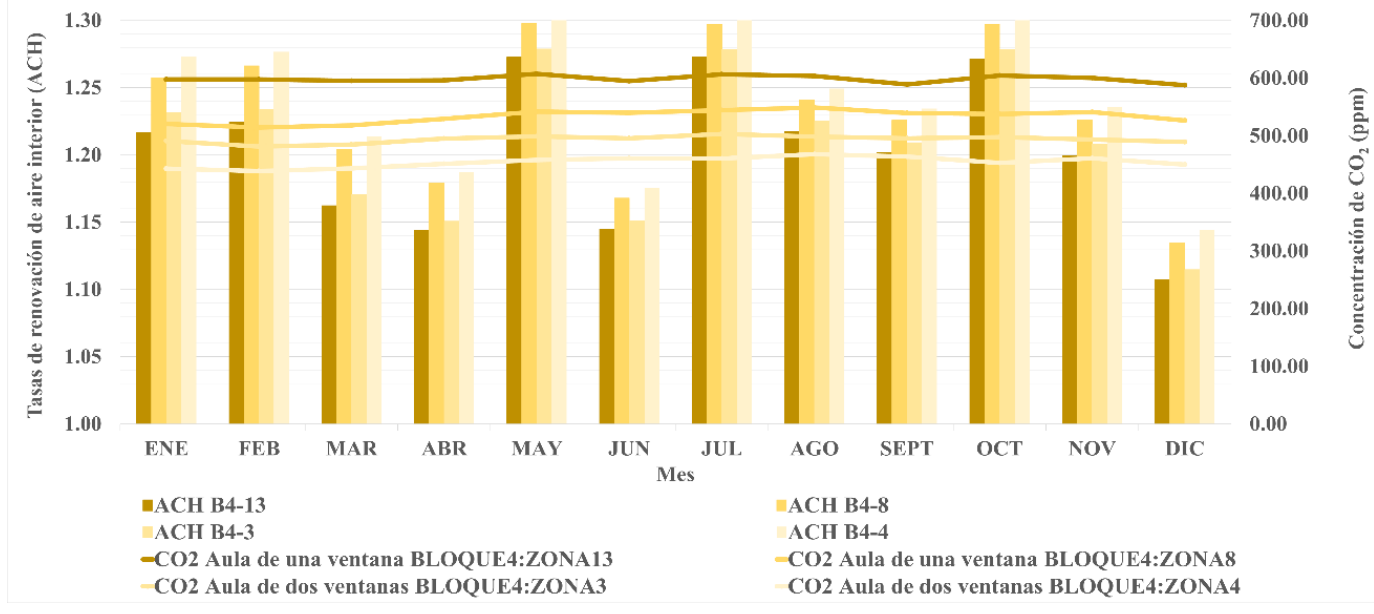

Figura 7: Comparación de tasas de renovación de aire interior con concentración de $\mathrm{CO}_{2}$. E5, tercer piso, modo pasivo. 


\section{Confort térmico}

En las figuras 8 y 9, se indican los resultados de las tasas de renovación de aire interior en comparación con el PMV para la planta baja y tercer piso, respectivamente. En el caso del PMV, los valores más cercanos a la sensación térmica neutral (" 0 ") fueron obtenidos en la planta baja del E5 (entre 0.60 a 0.40 , como se detalla en la figura 8 ) durante los meses de abril a diciembre. Durante enero, febrero y marzo, los valores de PMV alcanzan el valor de "1" que corresponde a la sensación térmica de "ligeramente caliente".

En contraste, el tercer piso registró valores entre "2" y "3" (Figura 9) durante todo el año, con sensación térmica "caliente" y "muy caliente". Los salones con una sola ventana ubicados en la fachada tanto en barlovento y en contra del viento registraron los mayores valores de PMV mientras que los salones con dos ventanas, los valores más bajos. Comparando estos datos con los valores más altos de las tasas de renovación interior, se destacó que a medida que aumentan los ACH a su vez el PMV registrado también aumenta, produciendo sensación térmica de calor dentro de las aulas de clases.

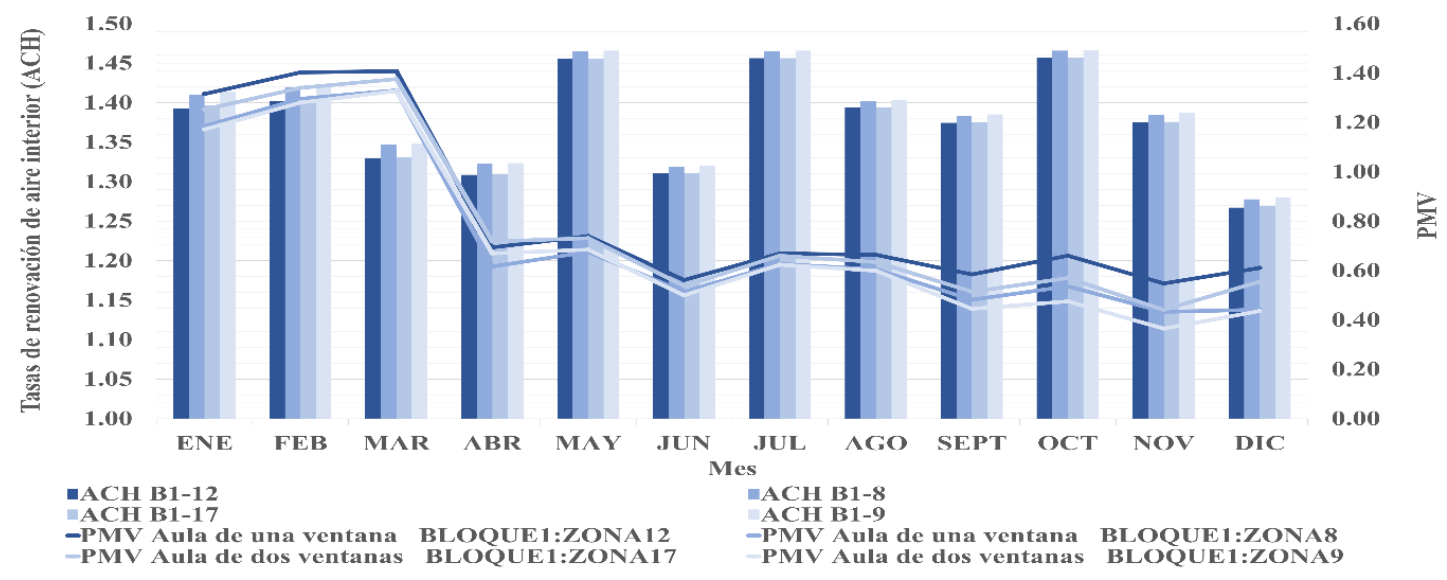

Figura 8: Comparación de tasas de renovación de aire interior con PMV. En planta baja de E5, modo pasivo.

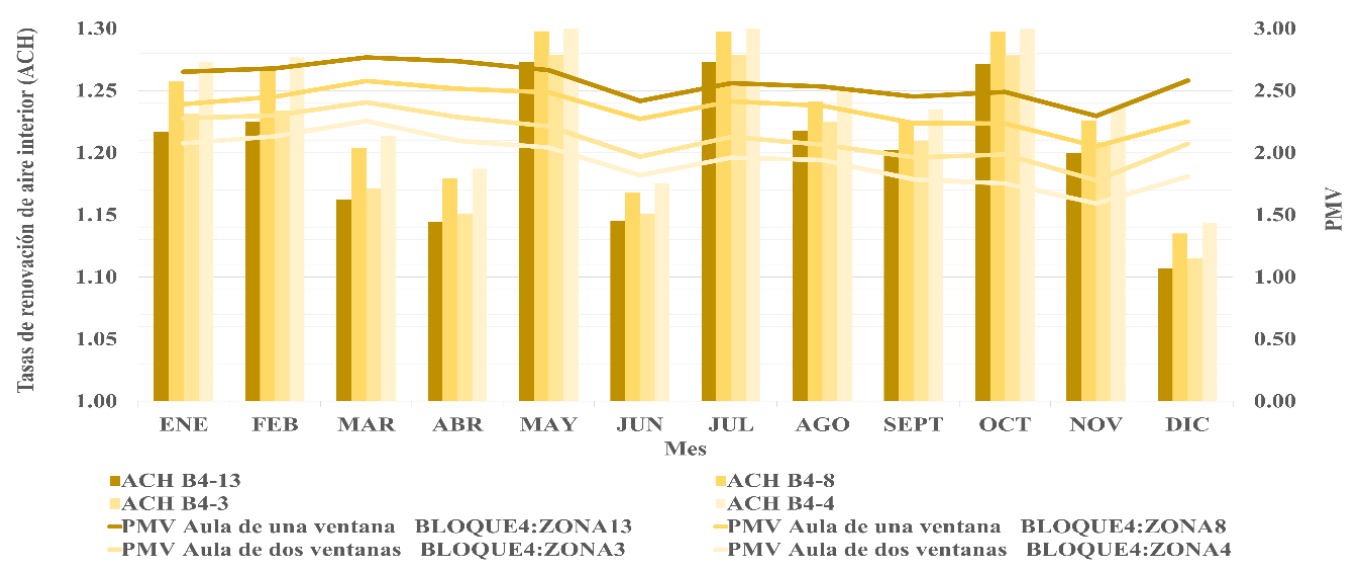

Figura 9: Comparación de tasas de renovación de aire interior con PMV en el tercer piso de E5, modo pasivo. 
En cuanto al modelo adaptativo al 80\% de aceptabilidad, ninguna de las plantas evaluadas (Figuras 10 y 11) del E5 obtiene un valor de "1". En el caso de la planta baja, durante los meses de enero, febrero, marzo, abril y diciembre los resultados obtenidos fueron debajo de " 0 " lo cual no resultan válidos para el estudio. En el caso del tercer piso, sucede lo mismo a lo largo de todo el año. Dicho esto, los valores aceptados por el modelo indicaron que los estudiantes a lo largo del año demostraron inconformidad térmica a lo largo del año.

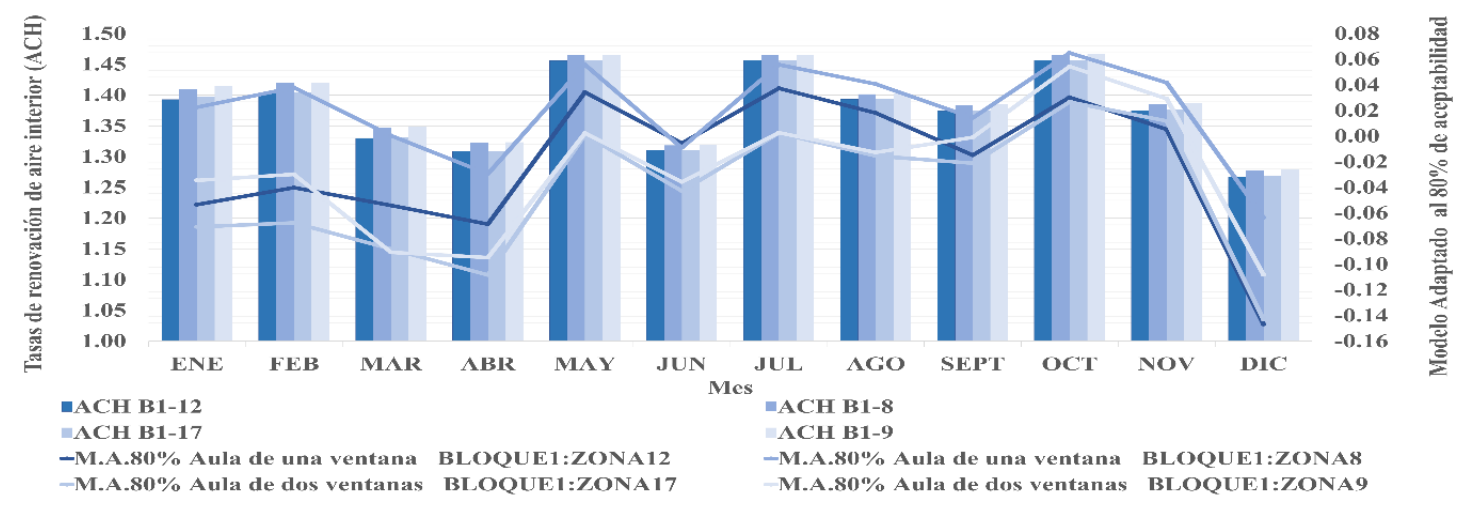

Figura 10: Comparación de tasas de renovación de aire interior con modelo adaptado al 80\% de aceptabilidad térmica. En la planta baja de E5, modo pasivo.

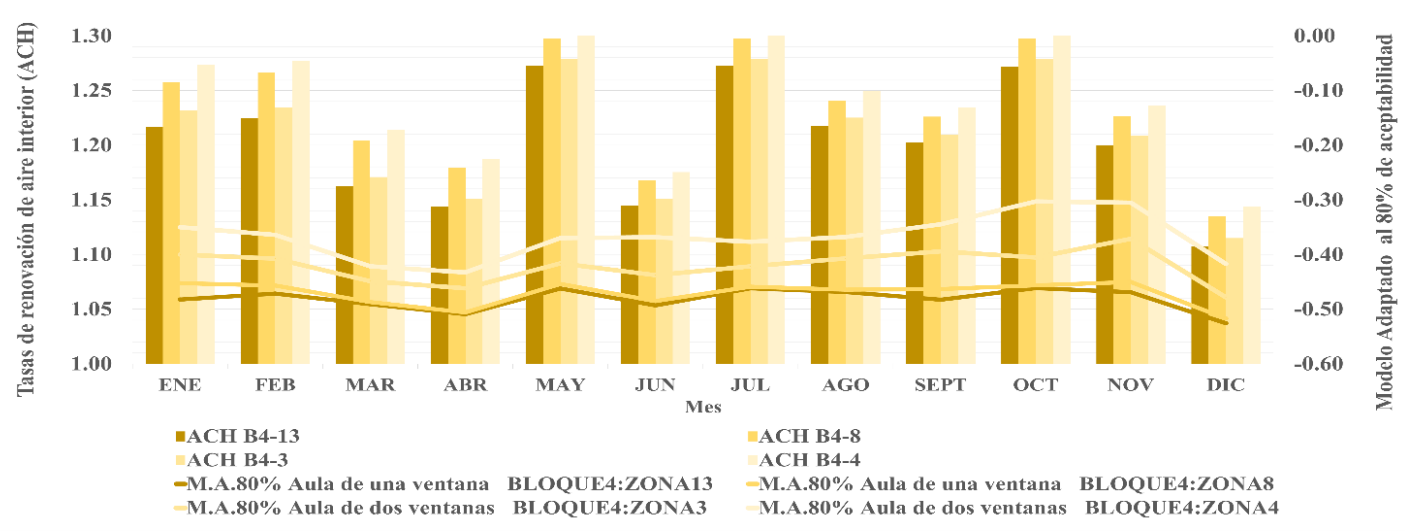

Figura 11: Comparación de tasas de renovación de aire interior con modelo adaptado al 80\% de aceptabilidad térmica. En el tercer piso de E5, modo pasivo.

\section{Edificio No. 1: calidad del aire}

En la planta baja se registró una máxima tasa de renovación de aire interior de $0.21 \mathrm{ACH}$ en el ala de laboratorios posterior del edificio (Figura 12). El resto de las aulas mantuvieron valores por debajo de 0.17 ACH. En el tercer piso (Figura 13), las tasas de renovación oscilaban entre 0.11 a 1.54 $\mathrm{ACH}$. El aula con mayor tasa de ventilación fue aquella ubicada en el ala de laboratorios posterior del edificio, llegando a 2.0 ACH. Ninguna de las plantas, cumplieron con el mínimo de $4.32 \mathrm{ACH}$ calculado previamente con la ecuación (1). Además, de las dos plantas estudiadas, las aulas con menores tasas de renovación de aire interior fueron aquellas en el ala de oficinas (parte central del edificio) y aquellas ubicadas en el ala de salones. Las mayores concentraciones de $\mathrm{CO}_{2}$, se registraron en aquellas aulas con menores tasas de ventilación. En el caso de la planta baja (Figura 12), la concentración en todos los salones superó los 1000 ppm; en el ala de salones incluso alcanza 3875 
ppm en el mes de agosto. La menor concentración, se obtuvo en las aulas ubicadas en el ala posterior de laboratorios, con un valor mínimo de 1440 ppm. En el tercer piso, hubo un registro de concentraciones entre 760 ppm a 1888 ppm. Las aulas ubicadas en el ala de laboratorios (tanto las frontales como posteriores) fueron las que mantuvieron concentraciones por debajo de $1000 \mathrm{ppm}$, mientras que el ala de salones y el ala central, superaron los 1000 ppm a lo largo del año (Figura 13).

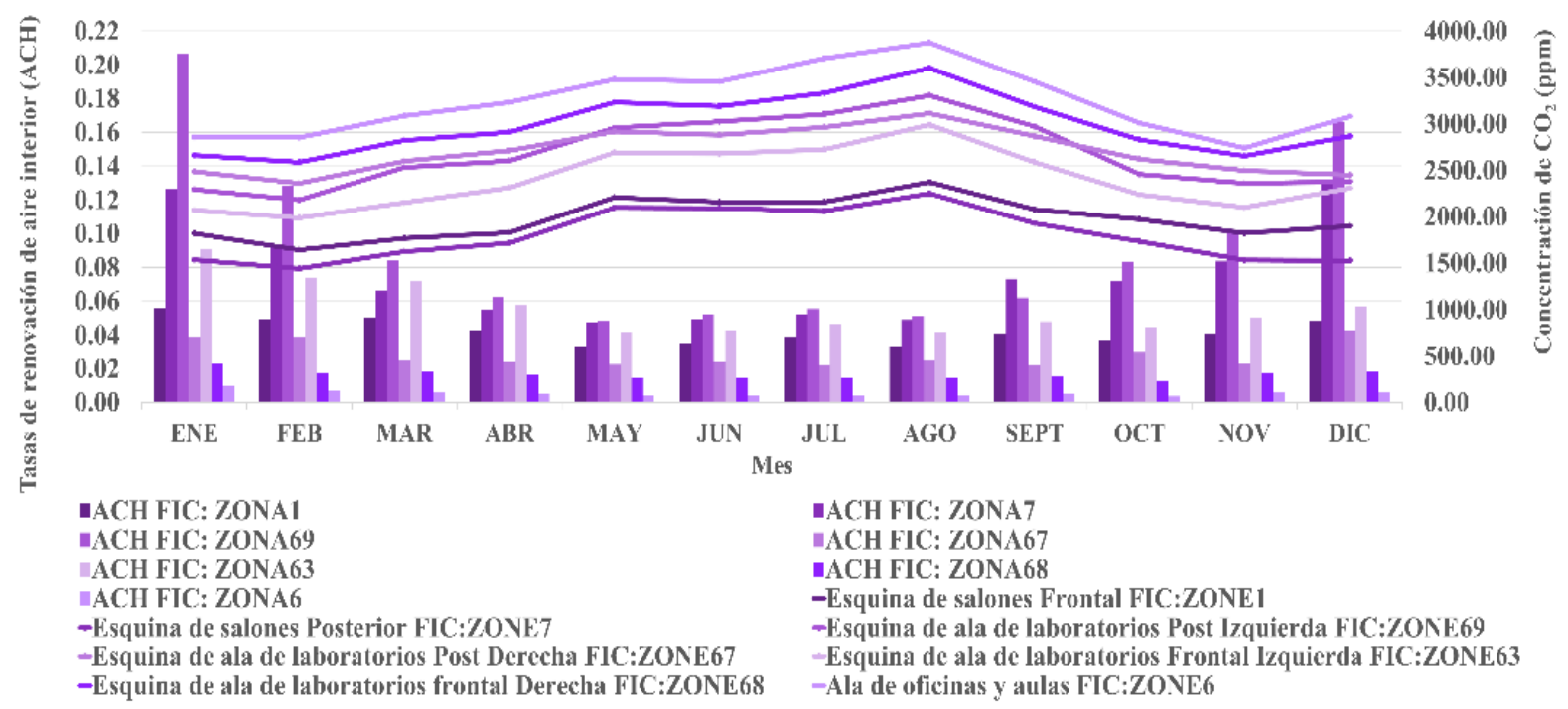

Figura 12: Comparación de tasas de renovación de aire interior con concentración de $\mathrm{CO}_{2}$ en la planta baja de E1, modo pasivo.

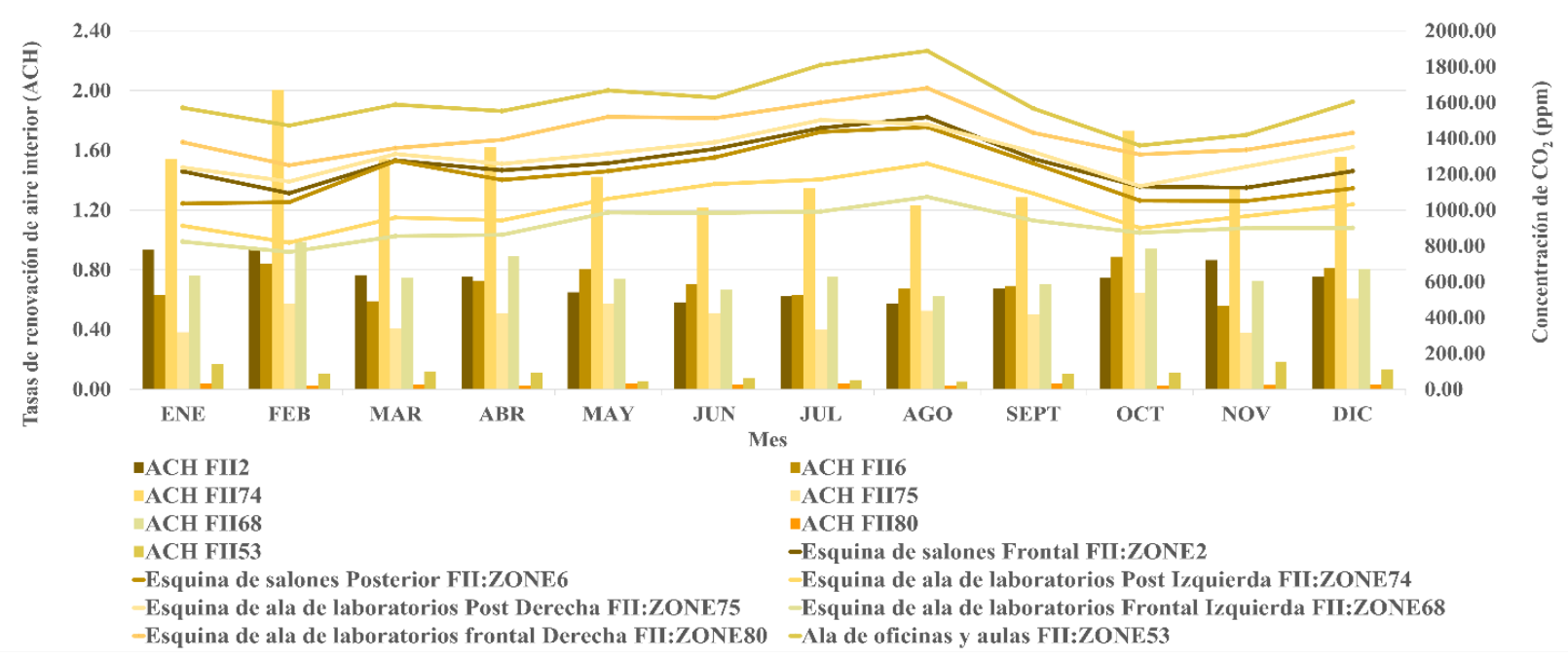

Figura 13: Comparación de tasas de renovación de aire interior con concentración de $\mathrm{CO}_{2}$ en el tercer piso de E1, modo pasivo.

\section{Confort térmico}

Los gráficos que comparan el PMV con la tasa de renovación del aire interior del modo pasivo del E1, reflejan un comportamiento proporcional: a medida que los ACH aumentan, el PMV aumenta y a medida que los $\mathrm{ACH}$ disminuyen, el PMV también. Las aulas que registraron los mayores valores de PMV, fueron aquellas con las mayores tasas de ventilación, es decir, las aulas de la parte posterior del edificio; mientras que las aulas con valores cercanos a la sensación térmica “neutral", fueron aquellas ubicadas en el ala de salones y área central del E1. 
En lo que respecta a la evaluación de la planta baja (Figura 14) los valores del PMV oscilaron desde -0.62 (sensación térmica "neutral" próximo a "ligeramente fresco") a 1.22 ("ligeramente caliente"). Durante el mes de enero, febrero y marzo, que corresponden a los meses de la estación seca, se registraron los valores más altos de PMV y el resto del año, que constituye la estación lluviosa, los más bajos; exceptuando octubre, noviembre y diciembre que forman parte del inicio de la estación seca.

Los recintos universitarios del tercer piso (Figura 15) alcanzaron datos de PMV desde 2.20 (caliente) a 3 (muy caliente), siendo los meses de enero, febrero, marzo, abril, noviembre y diciembre los meses con resultados registrados como sensación térmica "muy caliente". Esto, refleja un comportamiento similar al E5, donde al incrementar los pisos del edificio, la incomodidad térmica de los ocupantes se evidencia.

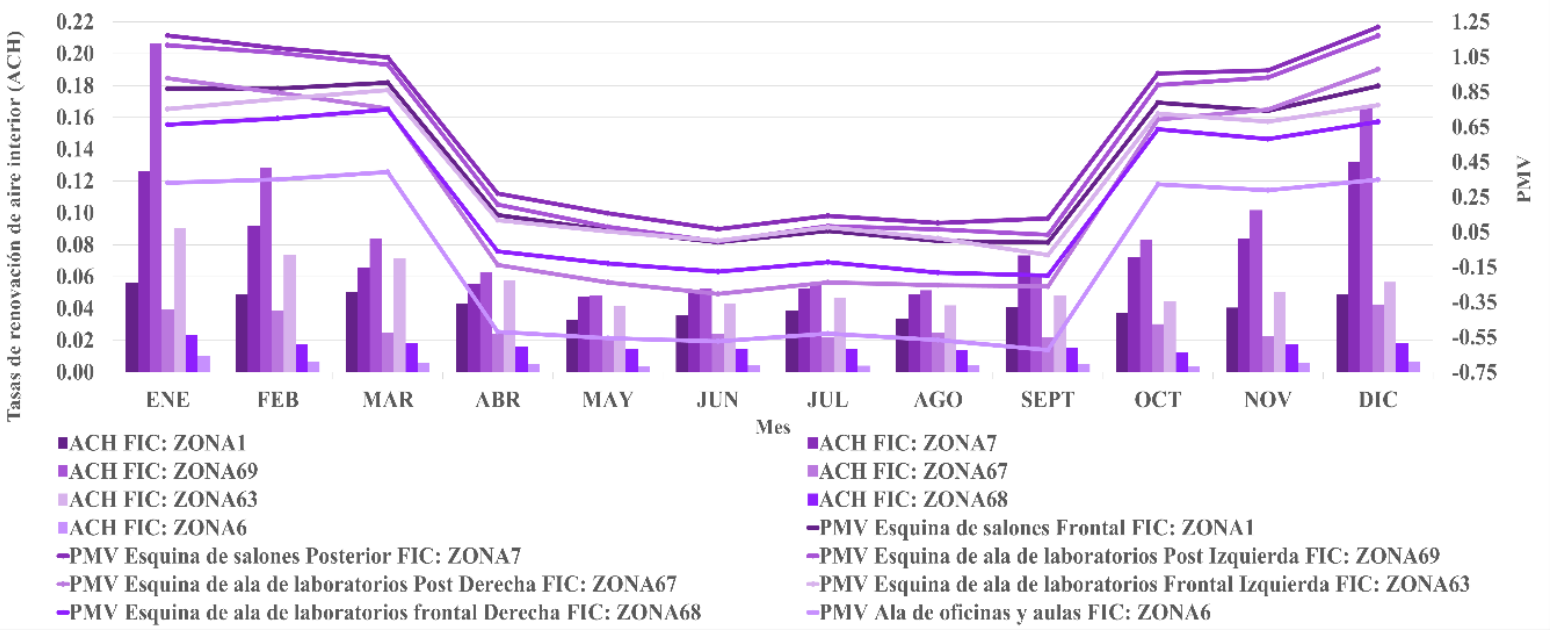

Figura 14: Comparación de tasas de renovación de aire interior con PMV. En la planta baja de E1, modo pasivo.

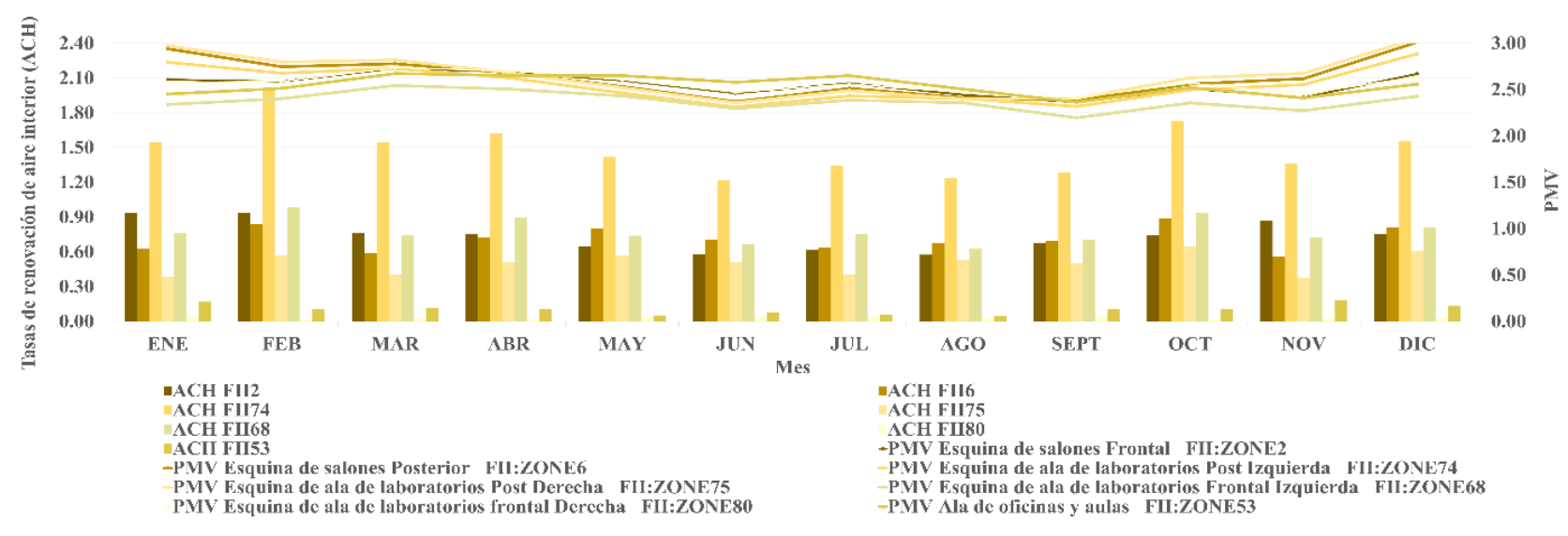

Figura 15: Comparación de tasas de renovación de aire interior con PMV. En el tercer piso de E1, modo pasivo.

En relación con el modelo adaptativo al $80 \%$ de aceptabilidad los resultados reflejaron un comportamiento semejante al E5, donde a lo largo de todo el año la planta baja y el tercer piso registraron datos inválidos negativos (Figura 16 y 17). 


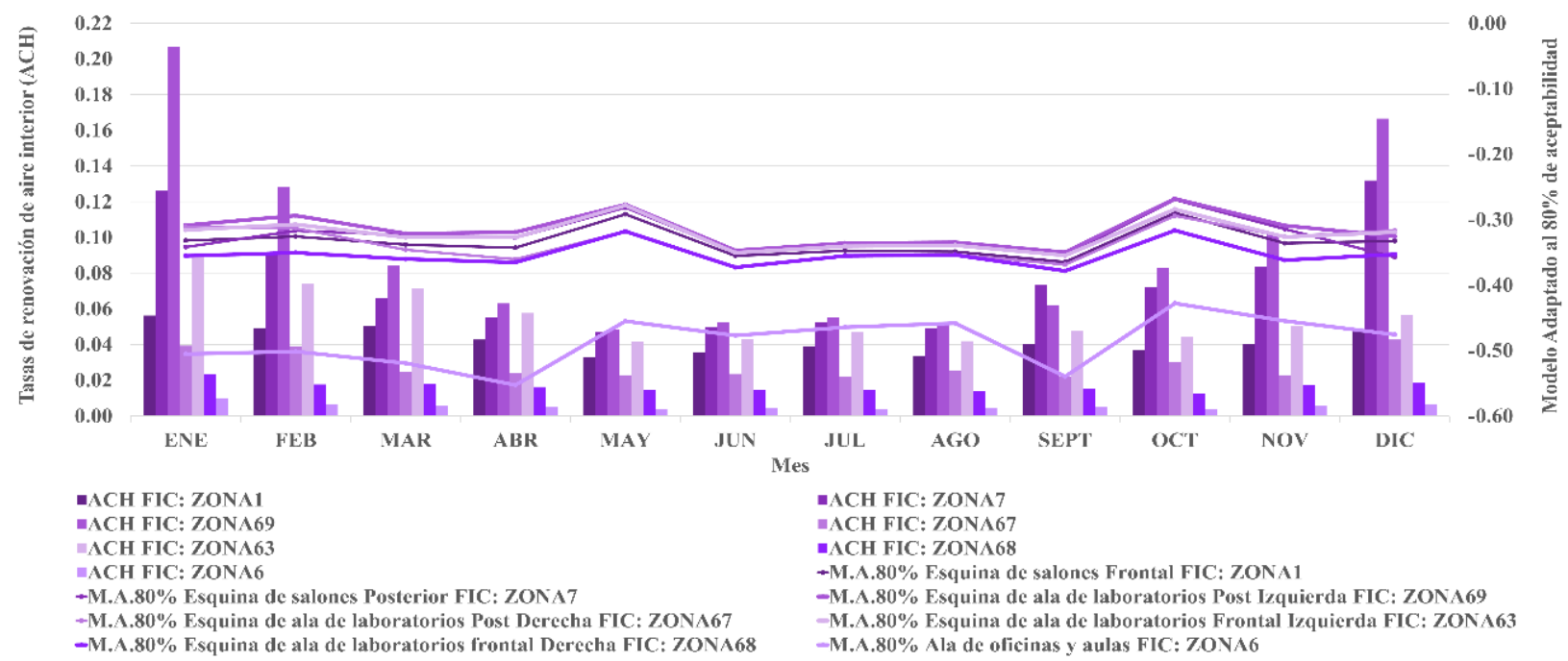

Figura 16: Comparación de tasas de renovación de aire interior con modelo adaptado al 80\% de aceptabilidad térmica. En la planta baja de E1, modo pasivo.

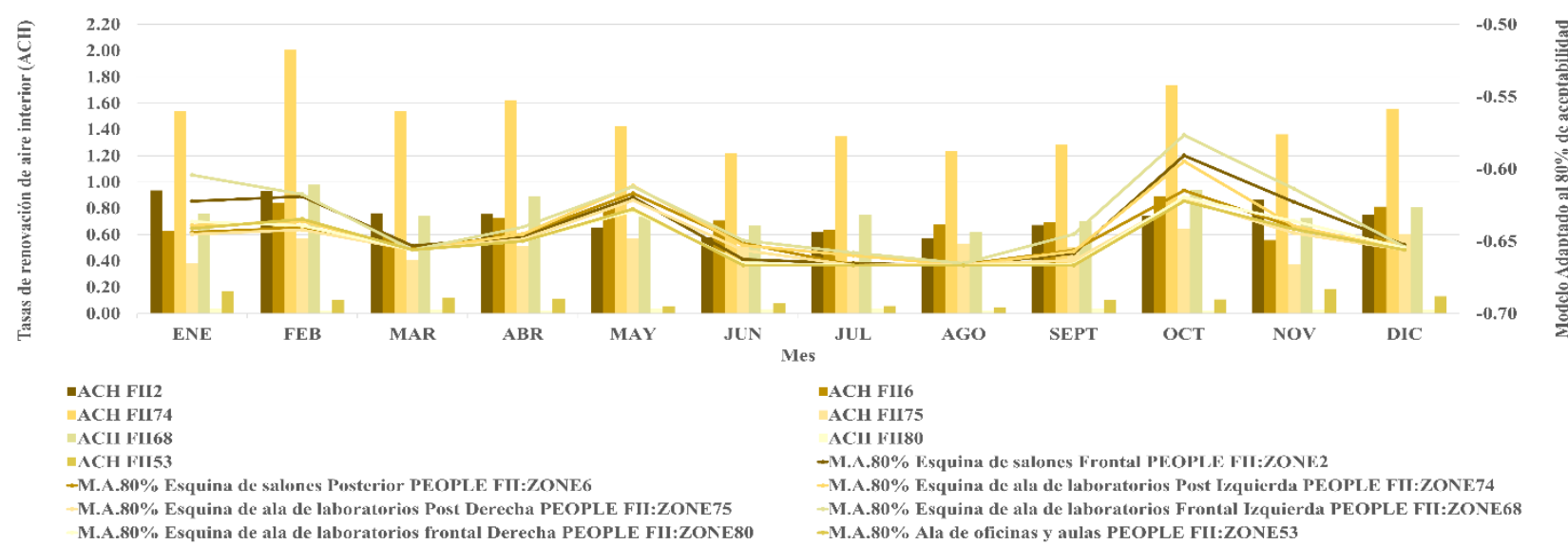

Figura 17: Comparación de tasas de renovación de aire interior con modelo adaptado al 80\% de aceptabilidad térmica. En el tercer piso de E1, modo pasivo.

Evaluación del sistema de ventilación mecánica, modo activo

Edificio No. 5: calidad del aire

El E5 en modo mecánico, al igual que en modo pasivo, no cumple con el mínimo de ventilación estimado de $5.13 \mathrm{ACH}$. Los valores de las aulas en planta baja, se mantuvieron entre 1.25 a 1.45 ACH (Figura 18), a diferencia del tercer piso cuyos salones de clases registraron valores entre 1.1 a $1.27 \mathrm{ACH}$ (Figura 19). En cuanto a la concentración de $\mathrm{CO}_{2}$, los niveles registrados superaron el límite de 1000 ppm. Las aulas de planta baja obtuvieron menores concentraciones de $\mathrm{CO}_{2}$ con niveles entre 2200 a 3143 ppm (Figura 18); a diferencia del tercer piso, las concentraciones se mantuvieron desde 2200 a 3800 ppm (Figura 19). 


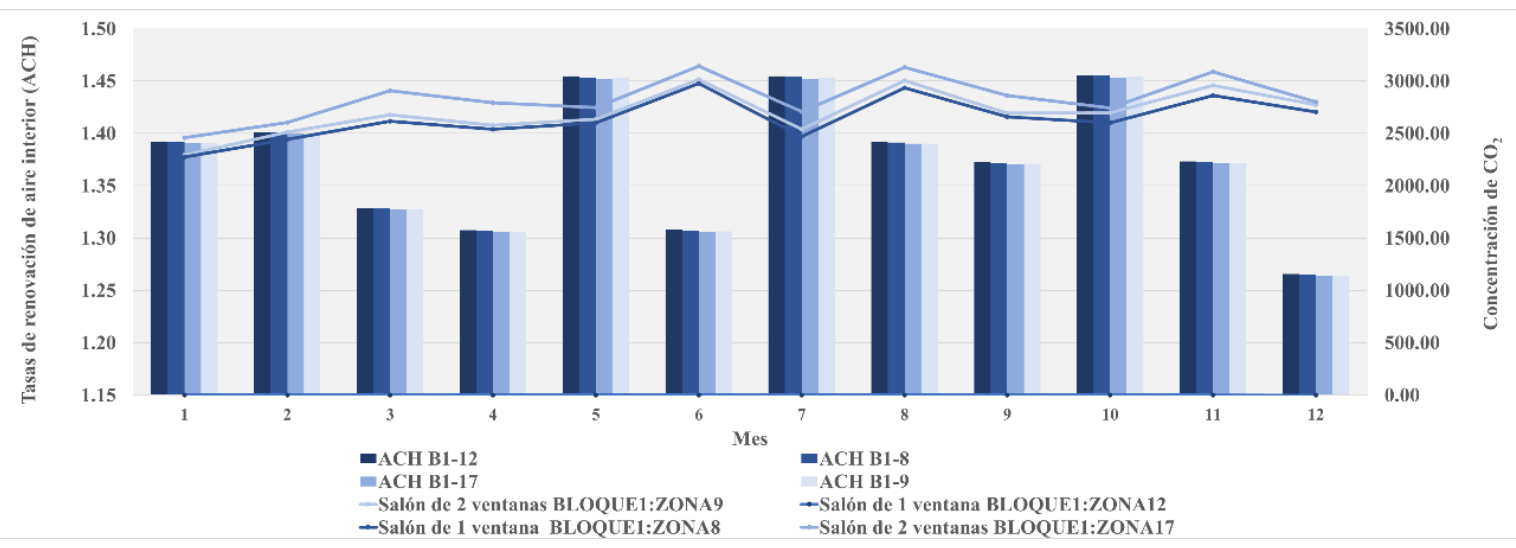

Figura 181: Comparación de tasas de renovación de aire interior con concentración de $\mathrm{CO}_{2}$. En la planta baja de E5, modo mecánico.

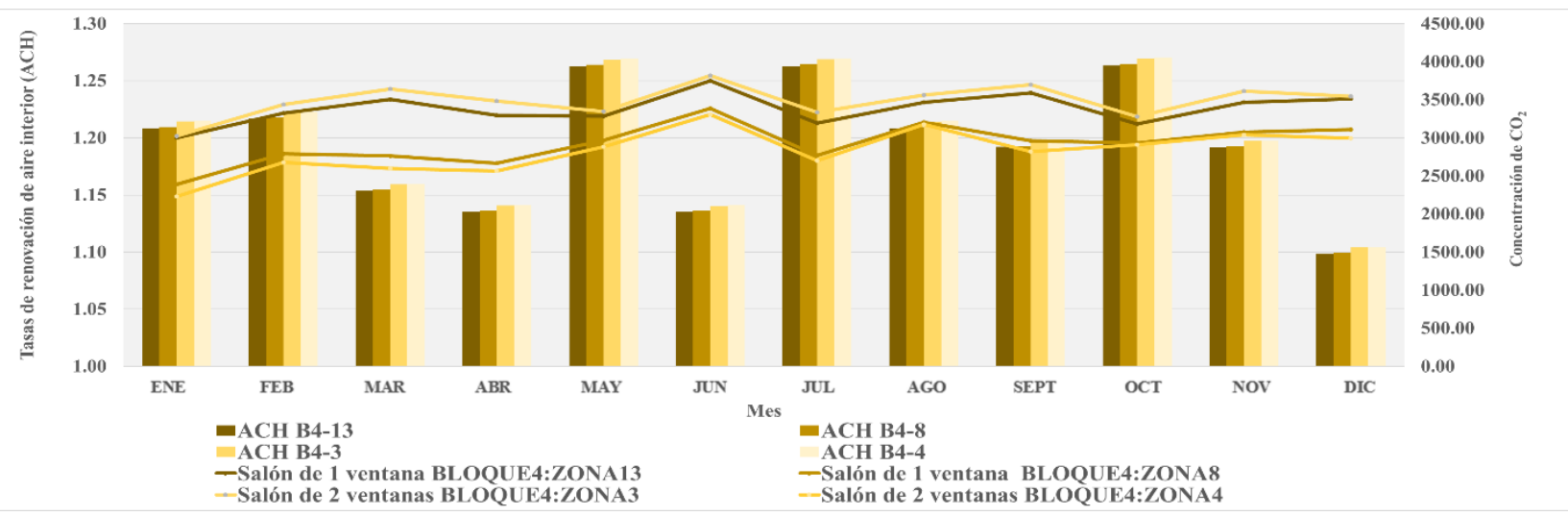

Figura 19: Comparación de tasas de renovación de aire interior con concentración de $\mathrm{CO}_{2}$. En el tercer piso de E5, modo mecánico.

\section{Confort térmico}

Durante los meses de enero, febrero y marzo, los valores de PMV se mantuvieron más altos a comparación del resto del año. Los ocupantes de la planta baja (Figura 20) a lo largo de los meses anteriores, reflejaron PMV entre 0.46 a 0.65 . Estos resultados se traducen como sensación térmica neutral/cómoda (0) y ligeramente caliente (1). Los ocupantes del tercer piso, (Figura 21) registraron valores entre 1.38 a 1.50. Los resultados anteriores, coinciden con la estación "seca" del país (enero y febrero). El resto del año, el voto medio previsto baja considerablemente coincidiendo con la estación lluviosa.

En el caso de la planta de los estudiantes de la planta baja, el PMV desde abril a diciembre osciló entre 0.25 a -0.5; esto se interpretó como sensación térmica "neutral". En cambio, los valores del voto medio previsto de los alumnos del tercer piso, indicaron sensación térmica "ligeramente caliente" (PMV entre 0.83 a 1.0). 


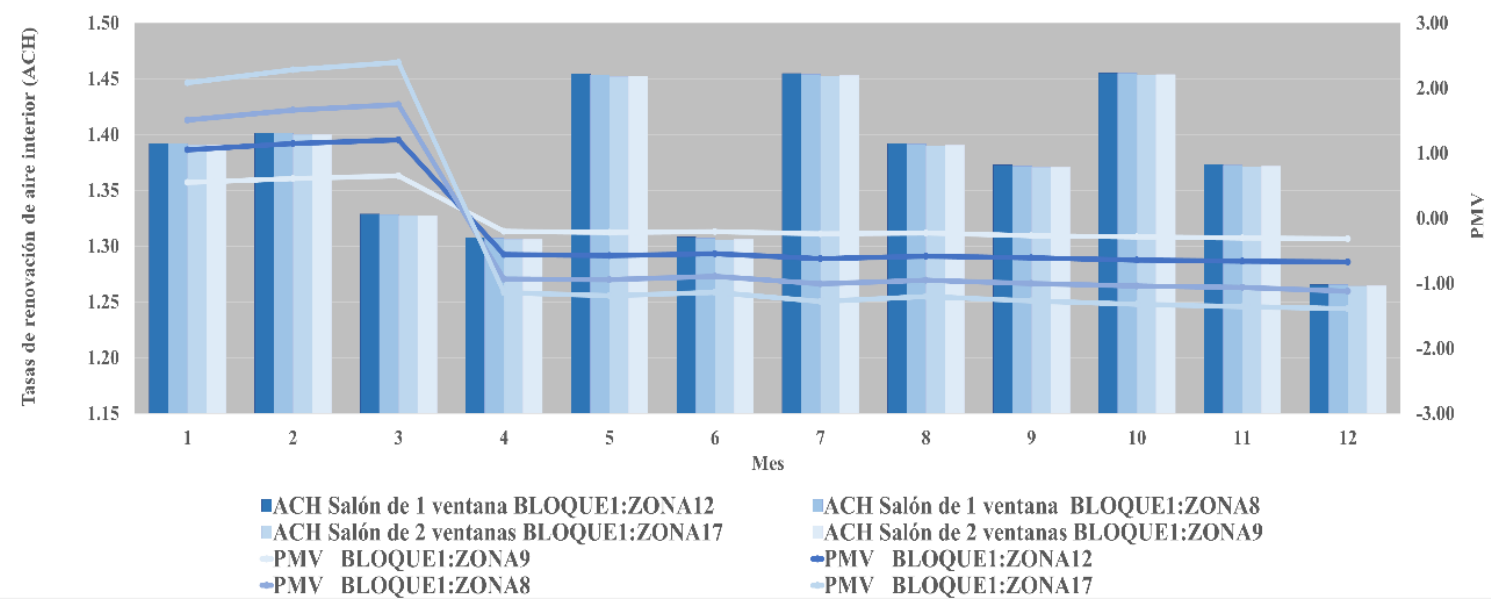

Figura 20: Comparación de tasas de renovación de aire interior con PMV. En la planta baja de E5, modo mecánico.

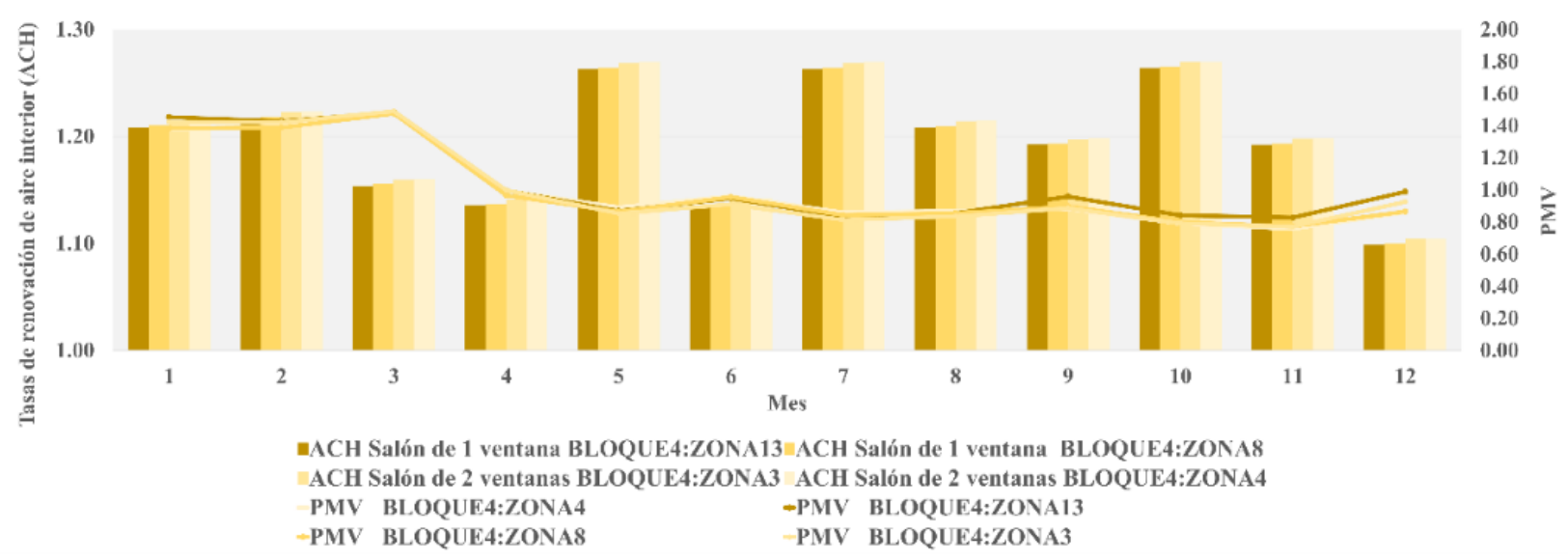

Figura 21: Comparación de tasas de renovación de aire interior con PMV. En el tercer piso de E5, modo mecánico.

Edificio No. 1: calidad del aire

Al igual que los resultados del modo pasivo, los salones de la planta baja (Figura 22) y del tercer piso (Figura 23) no cumplieron con el mínimo establecido de tasa de renovación de aire interior (4.32 ACH).

En lo que respecta a las aulas del primer piso, se registraron tasas de renovación entre 0.25 a 0.31 ACH con concentración de $\mathrm{CO}_{2}$ entre 960 y 1092 ppm (Figura 22), en tanto el tercer piso entre 0.25 a $0.39 \mathrm{ACH}$ con datos de $\mathrm{CO}_{2}$ entre 813 y 1050 ppm (Figura 23). A diferencia del resultado en modo pasivo, no existe una relación evidente entre las tasas de ventilación y la concentración de $\mathrm{CO}_{2}$ en los salones ya que varía de forma aleatoria a lo largo del año en modo activo. El sistema de ventilación mecánica en el E1 proporcionó una mejor calidad de aire interior ya que la concentración de $\mathrm{CO}_{2}$ en las distintas aulas, no superan 1100 ppm. 


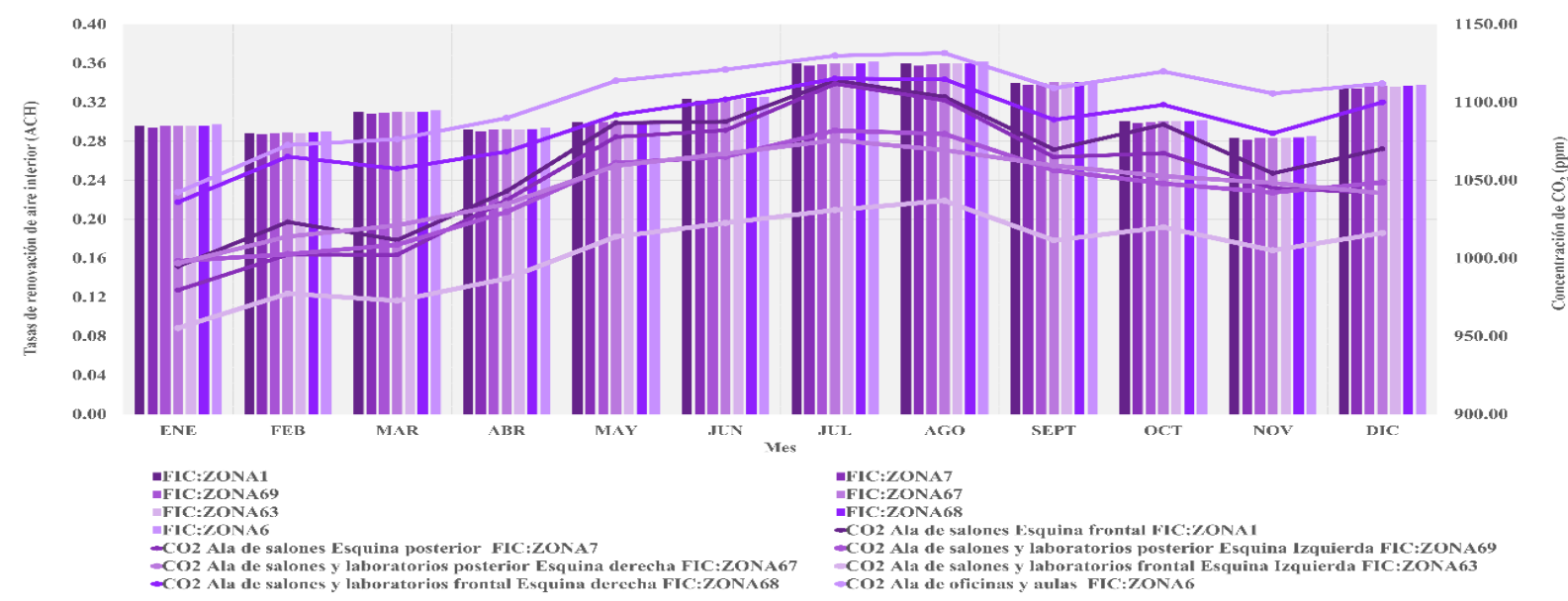

Figura 22: Comparación de tasas de renovación de aire interior con concentración de $\mathrm{CO}_{2}$. En la planta baja de E1, modo mecánico.

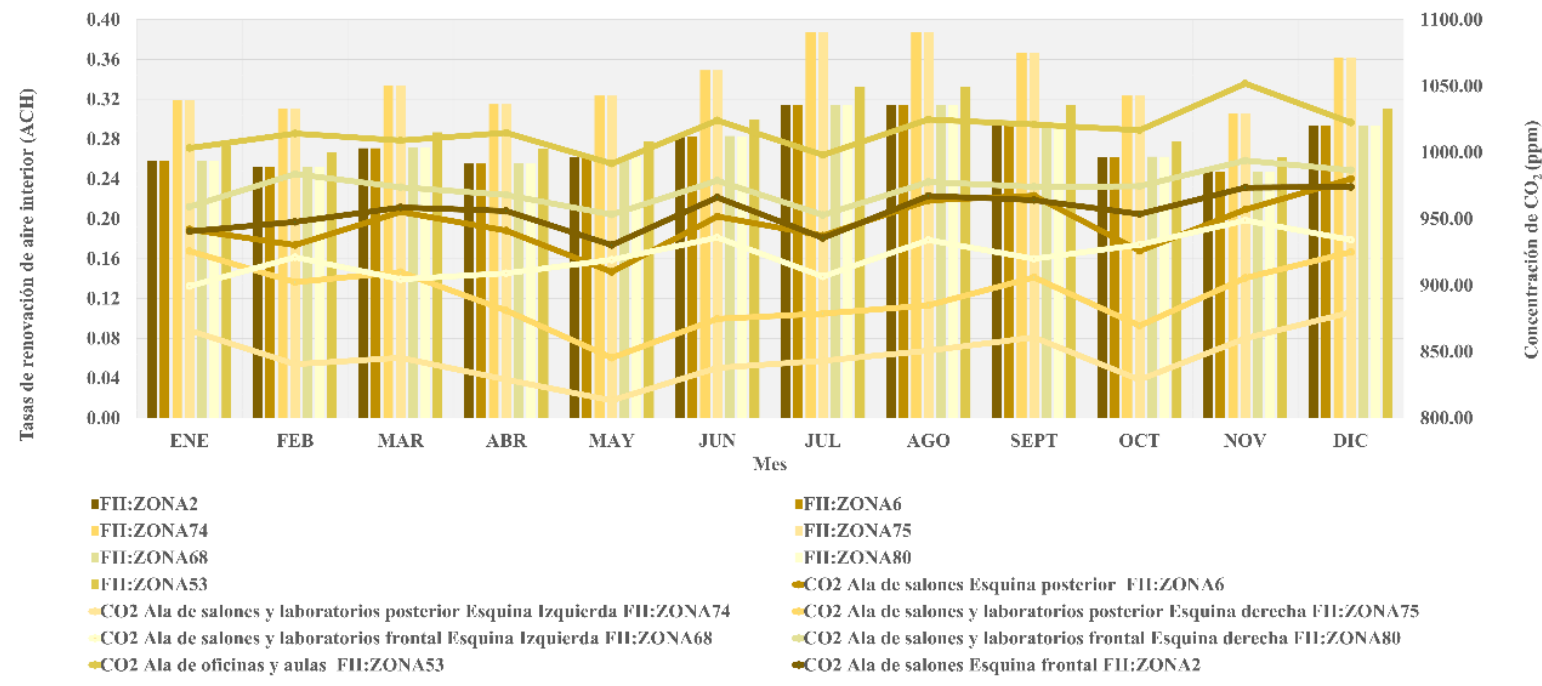

Figura 23: Comparación de tasas de renovación de aire interior con concentración de $\mathrm{CO}_{2}$. En el tercer piso de E1, modo mecánico.

\section{Confort térmico}

Semejante a los resultados obtenidos en el modo mecánico del E5, el PMV en ambas plantas del E1 resultaron más alto durante los meses de enero, febrero y marzo. Disminuyó desde abril, mayo, junio, julio, agosto hasta septiembre. A lo largo de octubre, noviembre y diciembre el voto medio previsto aumentó. En el caso de la planta baja (Figura 24), el PMV durante los meses de enero, febrero, marzo, octubre, noviembre y diciembre se mantuvo entre 0.28 a 0.67 ; una puntación intermedia entre la sensación térmica neutral (correspondiente a " 0 ") y ligeramente caliente (" 1 "). Durante los meses de estación lluviosa, el PMV resultante estuvo entre -0.33 a -0.69 , correspondiendo a un resultado intermedio entre neutral ("0") y ligeramente fresco (" 1 "). Finalmente, el tercer piso (Figura 25) obtuvo un PMV entre 0.92 a 1.91 durante los meses de estación seca y el resto del año un PMV entre 0.26 a 0.83 . 


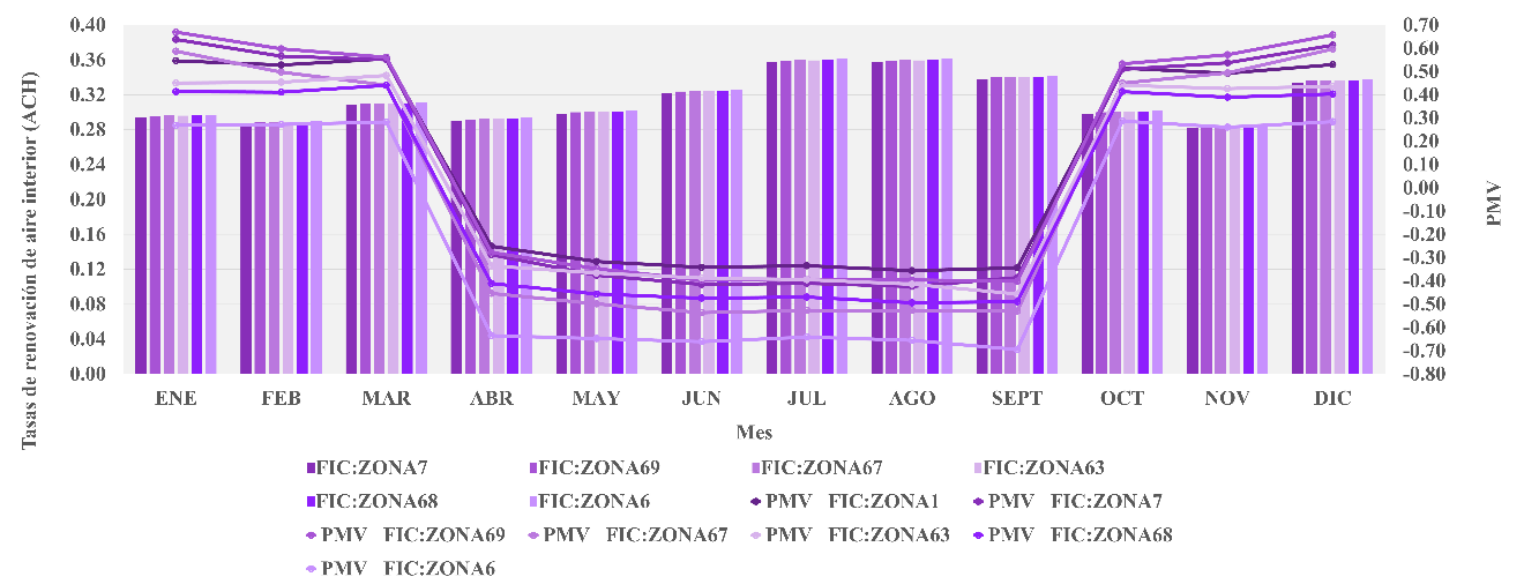

Figura 24: Comparación de tasas de renovación de aire interior con PMV. En la planta baja de E1, modo mecánico.

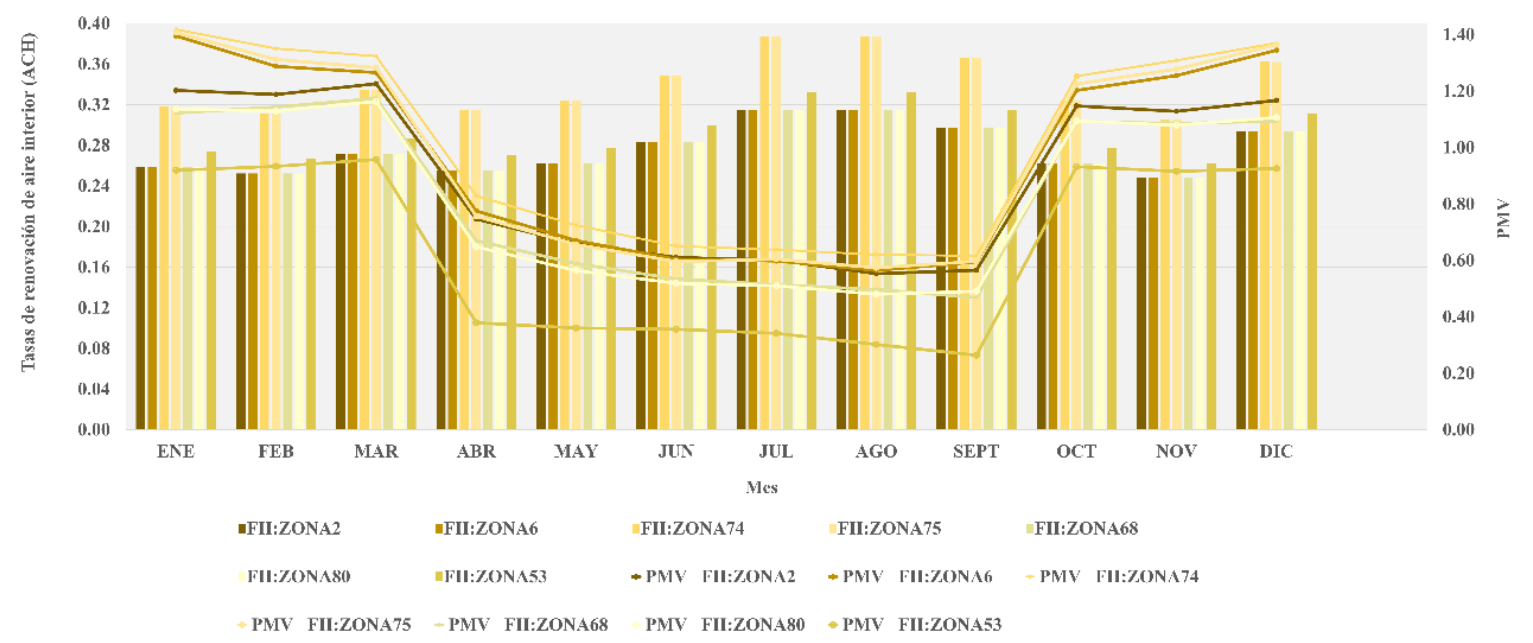

Figura 25: Comparación de tasas de renovación de aire interior con PMV. En el tercer piso de E1, modo mecánico.

\section{Discusión}

Modo pasivo: evaluación de la ventilación natural

La ventilación natural, es considerada como la solución más rápida y viable para asegurar ambientes interiores seguros, saludables y agradables térmicamente (Ji, Lomas, $\mathcal{E}$ Cook, 2009). Sin embargo, está condicionada por el clima donde esté ubicada la edificación, la envolvente que compone la estructura y el comportamiento humano (Atkinson et al., 2009). En general, en modo pasivo los resultados no fueron favorables para ningún edificio, ya que no se alcanzaron niveles mínimos apropiados de tasa de renovación de aire interior $(\mathrm{ACH})$ y confort térmico requeridos por los estándares 62.1 y 55.0, respectivamente (ASHRAE, 2019, 2020). Similarmente, Batterman et al. (2017), Calama-González et al. (2019) y Vartires et al. (2018), afirmaron que la apertura de ventanas, suele ser una opción económica y útil para favorecer el movimiento del aire dentro de espacios, pero resulta insuficiente para garantizar condiciones óptimas para una alta calidad del aire interior.

En el caso de las aulas que constituyen el E5, los resultados en términos de calidad del aire interior fueron admisibles para los salones de clase del tercer piso al no sobrepasar más de 1000 ppm a lo 
largo del año. Los resultados de ventilación natural de Ma'bdeh et al. (2020), realizados en aulas ubicadas en segundo y tercer piso, reflejan también concentraciones menores en aproximados de 600 ppm. Además, la diferencia de $\mathrm{CO}_{2}$ entre la planta baja y tercer piso de los dos edificios es significativa ya que, en ambos casos, las aulas de la planta baja obtuvieron los mayores valores de $\mathrm{CO}_{2}$ y el tercer piso, los menores valores. Esto es posible debido a la diferencia de altura entre las plantas, es decir, el aire caliente de la planta baja al ser menos denso tiende a ascender y esto provoca la creación de corrientes de aire fresco entre el aire exterior y el aire interior, producto de la diferencia de temperaturas del tercer piso del E1 y E5. Los salones de clase con dos ventanas del E5, registraron las menores concentraciones de dióxido de carbono al tener más espacio para el intercambio de aire a comparación de los salones con una sola ventana. En el E1 no se pudo hacer esta observación ya que todas las ventanas son del mismo tamaño.

Respecto al confort térmico, los indicadores evaluados reflejaron una sensación térmica "ligeramente caliente" en el E1 y "caliente" en el E5, mostrando así la inconformidad térmica con los resultados obtenidos del voto medio previsto. En el caso de la planta baja en ambos edificios, el confort térmico fue aceptable debido a que la sensación térmica variaba entre "neutral" y ligeramente caliente, pero, la calidad del aire ofrecida era evidentemente pobre al superar los 2000 ppm a lo largo del año. Resultados semejantes fueron publicados por Wargocki et al. (2020) quienes confirman que el uso exclusivo de ventilación natural no fue suficiente para mejorar la calidad del aire interior en aulas cuyas concentraciones superaban las 2100 ppm.

Se puede señalar además que, durante los meses de enero, febrero, marzo, noviembre y diciembre, que corresponden a la estación seca de Panamá, se registraron los mayores valores del PMV. En este tiempo, el voto medio previsto tuvo un comportamiento directamente proporcional con las tasas de renovación de aire interior. Esto resulta coherente porque en Panamá el clima es tropical de sabanas y debido a esto, la temperatura exterior suele estar más alta que la temperatura del interior de los edificios por lo que es posible que debido al intercambio de temperaturas, aumente el sobrecalentamiento de los edificios en general.

También, al observar las Figuras 12, 13, 18 y 19, que comparan la tasa de renovación de aire interior con la concentración de $\mathrm{CO}_{2}$, se determina que las tasas de renovación van ligadas de forma inversamente proporcional al nivel de concentración de $\mathrm{CO}_{2}$ por lo que es necesario aumentar los ACH utilizando equipos mecánicos. De esta manera, la concentración de $\mathrm{CO}_{2}$ en cada aula pudiese alcanzar niveles aceptados por las normativas de referencia y se ofrezca una alta calidad del aire interior a los estudiantes ( Ascione et al., 2021; Calama-González et al., 2019; Vartires et al., 2018). Las concentraciones de dióxido de carbono encontradas, según la normativa española RITE (Ministerio de Industria, Energía y Turismo del Gobierno de España, 2013), no representa un peligro considerable para la salud; sin embargo, concentraciones superiores a 2000 ppm puede provocar fatiga, dolores de cabeza y problemas de concentración (síntomas del edificio enfermo). También, la exposición al $\mathrm{CO}_{2}$ puede aumentar el ritmo cardíaco y provocar náuseas (Wisconsin WisDHS, 2019). En contraste, con el uso de equipos electromecánicos, es posible alcanzar mejores condiciones ambientales en interiores (Ascione et al., 2021). 
Finalmente, si bien ambos edificios están constituidos por los mismos materiales (desde las paredes, ventanas y puertas), la orientación de cada uno determina en gran medida cómo se comporta la ventilación natural. La ventilación natural, resulta favorable siempre y cuando las condiciones climáticas sean tales que la temperatura del aire exterior sea inferior a la temperatura del aire interior y se pueda asegurar una alta calidad de aire interior, de lo contrario, el uso de ventilación mecánica es necesaria (ASHRAE, 2019).

Modo mecánico: evaluación de la ventilación con el uso de sistema de acondicionamiento de aire

La ventilación mecánica, es utilizada como una estrategia para garantizar las mínimas tasas de renovación interior para mantener una alta calidad del aire (Poza-Casado, Gil-Valverde, Meiss, $\mathcal{E}$ Padilla-Marcos, 2021). A pesar de sustituir el modo de ventilación en ambos edificios, la ventilación mecánica no fue suficiente para alcanzar las mínimas tasas de renovación de aire interior en el E1 y E5 sugeridas por ASHRAE (2019). No obstante, las aulas de ambas plantas del E1 cumplieron con los criterios de calidad del aire interior; incluso con tasas menores a $0.5 \mathrm{ACH}$ en la planta baja y el tercer piso. La concentración de dióxido de carbono permaneció en ambas plantas, debajo de las 1200 ppm a lo largo del año. De manera similar, Vartires et al. (2018) confirmó que, al incluir equipos electromecánicos para mejorar la ventilación en aulas, el dióxido de carbono disminuyó de 2500 ppm a un aproximado de 1200 ppm. Reportes semejantes fueron publicados por Ascione et al. (2021), Calama-González et al. (2019) y Wargocki et al. (2020). Las aulas del E5, no mostraron mejorías en su calidad del aire interior pues las concentraciones registradas eran mayores a 2400 ppm.

El confort térmico tuvo mejorías significativas con la implementación del sistema mecánico de ventilación. Los estudiantes del E1 mostraron con el PMV una sensación térmica "neutral" y "ligeramente caliente", a diferencia de los salones del E5 que, a pesar de una pobre calidad de aire interior, el PMV para la planta baja fue en su mayoría "neutral" mientras que el tercer piso "ligeramente caliente". Ascione et al. (2021) mencionó que al proveer ventilación mecánica el voto medio previsto se mantuvo en valores menores a 1 y consideró que esta diferencia es válida debido a que la sensación térmica forma parte de ambientes controlados moderadamente.

Con el análisis realizado para el modo mecánico, no se observó a detalle una relación directa o inversa entre las tasas de renovación interior con la concentración de $\mathrm{CO}_{2}$. Durante los meses de enero y febrero se observó una relación directa donde al aumentar los $\mathrm{ACH}$ la concentración de $\mathrm{CO}_{2}$ en las aulas también aumenta. A partir de marzo, no se observa un patrón entre las tasas de ventilación y el $\mathrm{CO}_{2}$ pues, varía en los distintos meses para las plantas de los edificios. Contrariamente al análisis del modo pasivo, no existe relación de concentración de $\mathrm{CO}_{2}$ con la cantidad de ventanas o puertas de las aulas, tampoco la posición de las aulas referente a la altura de cada edificio. Esto debido a que, en modo mecánico, se considera un funcionamiento totalmente forzado (Ascione et al., 2021; Batterman et al., 2017; Calama-González et al., 2019). 


\section{Conclusiones}

En la investigación presentada, se logró evaluar los sistemas de ventilación natural y mecánica en dos edificios universitarios de la Universidad Tecnológica de Panamá, considerando la calidad del aire interior, tomando como referencia las tasas de renovación de aire y la concentración del dióxido de carbono, como también el voto medio previsto y modelo adaptativo de Fanger para determinar el confort térmico de los estudiantes dentro de las aulas, considerando su nivel de actividad y vestimenta.

Del análisis realizado, la ventilación natural no proveyó condiciones ambientales saludables para los estudiantes; a lo largo de todo el año, los estudiantes manifestaron inconformidad térmica y las concentraciones de dióxido de carbono superaron los máximos establecidos por normativas internacionales. La ventilación natural basada exclusivamente en la apertura de puertas y ventanas resulta insuficiente para garantizar una alta calidad del aire interior, sin considerar otros parámetros que lo permitan. La ventilación en modo pasivo resulta favorable siempre y cuando existan las condiciones que propicien su uso: desde la arquitectura, los materiales, el entorno y las condiciones climáticas donde se ubique un edificio, pero también, se deben considerar el confort térmico de los estudiantes, teniendo en cuenta su tipo de actividad y las características de vestimenta. En casos donde se dificulte asegurar condiciones ambientales favorables para uso de la ventilación natural, es necesario el uso de ventilación mecánica.

En modo activo, hubo mejorías considerables en términos de confort térmico en ambos edificios. No obstante, la calidad del aire interior aumentó solo en las aulas del E1, mientras que, en el E5, no fue suficiente el uso de equipos electromecánicos para mejorar las condiciones ambientales. Es importante considerar en este sentido, la ocupación de un espacio. Las aulas de los edificios de la Universidad Tecnológica de Panamá ocupan alrededor de 40 estudiantes por grupo. Un espacio donde la densidad de ocupación es alta, puede influir significativamente a la calidad del aire interior ya que es conocido que la concentración de $\mathrm{CO}_{2}$ en espacios interiores es debido principalmente al metabolismo humano.

El uso de programas que utilizan dinámica computacional de fluidos (CFD) permitió abordar la complejidad de la ventilación natural, predecir la ventilación mecánica e incluso la respuesta de las personas dentro de edificaciones con indicadores de confort térmico. Sin embargo, se deben considerar muchos parámetros al momento de evaluar el desempeño de la ventilación con este tipo de programación. Se recomienda iniciar con la definición de las condiciones del entorno del edificio a evaluar, incluyendo la ubicación geográfica, incidencias solares a lo largo del año, dirección del viento, la dimensión de los espacios, materiales que componen la edificación, la cantidad de personas que recurren el lugar y el tipo de actividad que ejercen en el lugar de estudio. Tomar en cuenta los parámetros mencionados, permitirá resultados más precisos al momento de evaluar el desempeño de la ventilación en edificios de diversa índole. 


\section{Contribución de los autores}

En concordancia con la taxonomía establecida internacionalmente para la asignación de créditos a autores de artículos científicos (https://casrai.org/credit/). Los autores declaran sus contribuciones en la siguiente matriz:

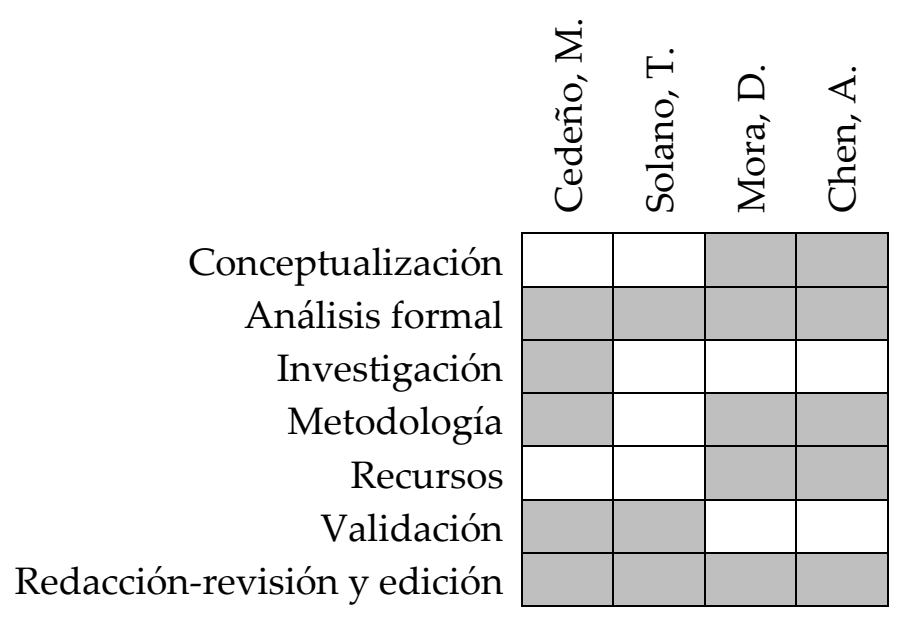

\section{Conflicto de Interés}

Los financiadores no tuvieron ningún papel en el diseño del estudio; en la recogida, análisis o interpretación de los datos; en la redacción del manuscrito o en la decisión de publicar los resultados.

\section{Agradecimientos}

Los autores agradecen a la Facultad de Ingeniería Mecánica de la Universidad Tecnológica de Panamá por su colaboración. Esta investigación fue financiada por la Institución Panameña secretaria nacional de Ciencia, Tecnología e Innovación (SENACYT) https://www.senacyt.gob.pa/, bajo los proyectos con códigos FID18-056 y FIED19-R2-005, así como del Sistema Nacional de Investigación (SNI).

\section{Referencias}

ASHRAE. (2009). Indoor Air Quality Guide: Best Practices for Design, Construction and Commissioning (Pap/Cdr ed.). Recuperado de: https://www.ashrae.org/technicalresources/bookstore/indoor-air-quality-guide

ASHRAE. (2019). ANSI/ASHRAE Standard 62.1-2019: Ventilation for Acceptable Indoor Air Quality. https://ashrae.iwrapper.com/ASHRAE_PREVIEW_ONLY_STANDARDS/STD_62.1 _2019

ASHRAE. (2020). ANSI/ASHRAE Standard 55-2020: Thermal Environmental Conditions for Human 
https://ashrae.iwrapper.com/ASHRAE_PREVIEW_ONLY_STANDARDS/STD_55_2 020

Ascione, F., De Masi, R. F., Mastellone, M., \& Vanoli, G. P. (2021). The design of safe classrooms of educational buildings for facing contagions and transmission of diseases: A novel approach combining audits, calibrated energy models, building performance (BPS) and computational fluid dynamic (CFD) simulations. Energy and Buildings, 230, 110533. https://doi.org/10.1016/j.enbuild.2020.110533

ASHRAE Epidemic Task Force. (2020). Laboratory Subcommittee Guidance Document. Recuperado de: https://www.ashrae.org/file\%20library/technical\%20resources/covid-19/ashrae-etf--lab-guidance.pdf

Atkinson, J., Chartier, Y., Pessoa-Silva, C.L., Jensen, P., Li, Y., Seto, W.-H., \& World Health Organization (2009). WHO Publications/Guidelines: Natural Ventilation for Infection Control in Health-Care Settings. Recuperado de: https://www.who.int/water_sanitation_health/publications/natural_ventilation.pdf

Bakó-Biró, Z., Clements-Croome, D. J., Kochhar, N., Awbi, H. B., E Williams, M. J. (2012). Ventilation rates in schools and pupils' performance. Building and Environment, 48(1), 215-223. https://doi.org/10.1016/j.buildenv.2011.08.018

Batterman, S., Su, F.-C., Wald, A., Watkins, F., Godwin, C., $\mathcal{E}$ Thun, G. (2017). Ventilation rates in recently constructed U.S. school classrooms. Indoor Air, 27(5), 880-890. https://doi.org/10.1111/ina.12384

Calama-González, C. M., León-Rodríguez, Á. L., \& Suárez, R. (2019). Indoor Air Quality Assessment: Comparison of Ventilation Scenarios for Retrofitting Classrooms in a Hot Climate. Energies, 12(24), 4607. https://doi.org/10.3390/en12244607

Cruz, M., Querol, X., Alastuey, A., Riediker, M., Felisi, J. M., Beko, G., ... Carslaw, N. (2020). Guide for ventilation towards healthy classrooms. Recuperado de: https://scoeh.ch/wp-content/uploads/2021/01/Guide-forventilation_Indairpollnet.pdf

D’Ambrosio, F., Bellia, L., Boerstra, A., Dijken, F. Van, Ianniello, E., Lopardo, G., ... Romagnoni, P. (2010). Environment and Energy Efficiency in Schools (Part 1), REHVA Guidebook Number vol. 13, REHVA, Brussels, Belgium. Recuperado de: https://www.rehva.eu/eshop/detail/indoor-environment-and-energy-efficiency-ineducational-buildings

DesignBuilder Software (2021). Design Building: Summary Outputs (KPIs). Recuperado de: https://designbuilder.co.uk/helpv6.0/Content/KPIs.htm

European Commitee for Standarization (2006). prEN 13779:2006: Ventilation for nonresidential buildings - Performance requirements for ventilation and room-conditioning 
systems.

Recuperado

de

http://www.cres.gr/greenbuilding/PDF/prend/set4/WI_25_Pre-

FV_version_prEN_13779_Ventilation_for_non-resitential_buildings.pdf

Fantozzi, F., E Rocca, M. (2020). An extensive collection of evaluation indicators to assess occupants' health and comfort in indoor environment. Atmosphere, 11(1), p. 90. https://doi.org/10.3390/atmos11010090

Fisk, W. J. (2017). The ventilation problem in schools: literature review. Indoor Air, 27(6), 1039-1051. https://doi.org/10.1111/ina.12403

Google Earth. (s. f.). Google Earth Universidad Tecnológica de Panamá. Recuperado de: https://earth.google.com/web/search/Universidad+Tecnol\%c3\%b3gica+de+Panam\% c3\%a1,+V\%c3\%ada+Centenario,+Panam\%c3\%a1/@9.02354187,$79.53286043,65.91719949 a, 450.7178551 d, 35 y,-$

21.18805442h,13.26082563t,0r/data=CigiJgokCTB9hRg1AzVAES99hRg1AzXAGaohK 5nFuUlAIachK5nFuUnA?hl=es

Hu, H. H. (2012). Computational Fluid Dynamics. In P. K. Kundu, I. M. Cohen \& D. R. Dowling (Eds.), Fluid Mechanics (pp. 421-472). Elsevier. https://doi.org/10.1016/B9780-12-382100-3.10010-1

Ji, Y., Lomas, K. J., \& Cook, M. J. (2009). Hybrid ventilation for low energy building design in south China. Building and Environment, 44(11), 2245-2255. https://doi.org/10.1016/j.buildenv.2009.02.015

Jiménez, A. (2021). UF0422: Mantenimiento correctivo de instalaciones de climatización y ventilación-extracción.

Recuperado

de: https://books.google.com.pa/books?id=RwIwEAAAQBAJE्Eprintsec=frontcover $\mathcal{E} h \mathrm{l}=$ es $\mathcal{E}$ source $=$ gbs_ge_summary_r $\mathcal{E}$ cad $=0 \# \mathrm{v}=$ onepage $\mathcal{E} \mathrm{q} \mathcal{E} \mathrm{f}=$ false

Junta Técnica de Ingeniería y Arquitectura (JTIA), E Asociación Panameña de Aire Acondicionado y Ventilación (APAYRE). (2014). Resolución 117 de 11 de diciembre de 2013: Por medio de la cual se aprueba el Reglamento de Aire Acondicionado y Ventilación para la República de Panamá. Recuperado de: https://www.asamblea.gob.pa/APPS/LEGISPAN/PDF_NORMAS/2010/2013/2013_6 08_1338.pdf

Ma'bdeh, S. N., Al-Zghoul, A., Alradaideh, T., Bataineh, A., E Ahmad, S. (2020). Simulation study for natural ventilation retrofitting techniques in educational classrooms - A case study. Heliyon, 6(10), e05171. https://doi.org/10.1016/j.heliyon.2020.e05171

Ministerio de Educación de Chile. (1989). Decreto 548: Aprueba Normas para la planta física de los locales educacionales que establecen las exigencias mínimas que deben cumplir los establecimientos reconocidos como cooperadores de la función educacional del Estado, según 
el nivel y modalidad de la enseñanza que impartan. Recuperado de: https://www.bcn.cl/leychile/navegar?idNorma=14166

Ministerio de Industria, Energía y Turismo del Gobierno de España. Comentarios RITE-2007: Reglamento de Instalaciones Térmicas en los Edificios. (2007). Recuperado de: https://www.idae.es/uploads/documentos/documentos_10540_Comentarios_RITE_ GT7_07_2200d691.pdf

Ministerio de Salud de Costa Rica. (2021). LS-SI-014: Lineamientos para sistemas de ventilación y aire acondicionado (COVID-19). Recuperado de: https://www.ministeriodesalud.go.cr/sobre_ministerio/prensa/docs/ls_si_014_linea mientos_sistemas_ventilacion_aire_acondicionado_03062021.pdf

Pita, E. (1994). Acondicionamiento de aire: Principios y sistemas de refrigeración. Recuperado de: https://es.scribd.com/document/509292129/Acondicionamiento-deAire-Edward-Pita-2da-Edicion

Poza-Casado, I., Gil-Valverde, R., Meiss, A., E Padilla-Marcos, M. Á. (2021). Impact of Air Infiltration on IAQ and Ventilation Efficiency in Higher Educational Classrooms in Spain. Sustainability, 13(12), 6875. https://doi.org/10.3390/su13126875

Pulimeno, M., Piscitelli, P., Colazzo, S., Colao, A., E Miani, A. (2020). Indoor air quality at school and students' performance: Recommendations of the UNESCO Chair on Health Education and Sustainable Development $\mathcal{E}$ the Italian Society of Environmental Medicine (SIMA). Health Promotion Perspectives, Vol. 10, pp. 169-174. https://doi.org/10.34172/hpp.2020.29

Simanic, B., Nordquist, B., Bagge, H., E Johansson, D. (2019). Indoor air temperatures, CO2 concentrations and ventilation rates: Long-term measurements in newly built lowenergy schools in Sweden. Journal of Building Engineering, 25(6), 100827. https://doi.org/10.1016/j.jobe.2019.100827

Sun, Y., Wang, Z., Zhang, Y., \& Sundell, J. (2011). In China, Students in Crowded Dormitories with a Low Ventilation Rate Have More Common Colds: Evidence for Airborne Transmission. PLoS ONE, 6(11), e27140. https://doi.org/10.1371/journal.pone.0027140

The American Institute of Architects (AIA). (2021). Risk Management Plan for Buildings AIA. (April). Recuperado de: https://www.aia.org/resources/6299432-riskmanagement-plan-for-buildings?editing=true $\mathcal{E}$ tools=true

Trebilcock, M., Soto, J., Figueroa, R., E Piderit-Moreno, B. (2016). Metodología para el diseño de edificios educacionales confortables y resilientes. AUS, (20), 70-76. https://doi.org/10.4206/aus.2016.n20-11

United States Environmental Protection Agency (EPA). (1991). Indoor Air Facts No. 4 Sick Building Syndrome. EPA - Air E Radiation (6609J), Research and Development (MD-56), 
56, 1-4. Recuperado de: https://www.epa.gov/sites/production/files/201408/documents/sick_building_factsheet.pdf

Vartires, A., Damian, A., Olariu, A., Istrate, A., Catalina, T., E Zorilă, E. (2018). Challenges in achieving a high indoor air quality in an educational building. Revista Romana de Inginerie Civila, 9(1), 28-42. Recuperado de: https://www.proquest.com/openview/59015e81a32a57ead207E51a45139f95/1.pdf?p q-origsite $=$ gscholar $\mathcal{E}$ cbl $=2029207$

Wargocki, P., Porras-Salazar, J. A., Contreras-Espinoza, S., E Bahnfleth, W. (2020). The relationships between classroom air quality and children's performance in school. $\begin{array}{lll}\text { Building and Environment, } & 106749 .\end{array}$ https://doi.org/10.1016/j.buildenv.2020.106749

Wargocki, P., E Wyon, D. P. (2013). Providing better thermal and air quality conditions in school classrooms would be cost-effective. Building and Environment, 59, 581-589. https://doi.org/10.1016/j.buildenv.2012.10.007

Williams, S. (2017). Welcome to Your World: How the Built Environment Shapes Our Lives. New York, EE.UU. HarperCollins.

Wisconsin WisDHS. (2019). Carbon Dioxide I Wisconsin Department of Health Services. Retrieved December 14, 2021, from Wisconsin Department of Health Services website: https://www.dhs.wisconsin.gov/chemical/carbondioxide.htm 